\title{
Prospects for a Muon Spin Resonance Facility in the MuCool Test Area
}

\author{
John A Johnstone
}

\begin{abstract}
This paper investigates the feasibility of re-purposing the MuCool Test Area beamline and experimental hall to support a Muon Spin Resonance facility, which would make it the only such facility in the US. This report reviews the basic muon production concepts studied and operationally implemented at TRIUMF, PSI, and RAL and their application to the MTA facility. Two scenarios were determined feasible. One represents an initial minimal-shielding and capital-cost investment stage with a single secondary muon beamline that transports the primary beam to an existing high-intensity beam absorber located outside of the hall. Another, upgraded stage, involves an optimized production target pile and high-intensity absorber installed inside the experimental hall and potentially multiple secondary muon lines. In either scenario, with attention to target design, the MTA can host enabling and competitive Muon Spin Resonance experiments.
\end{abstract}

\section{Introduction}

The MuCool Test Area (MTA) is an experimental area that was designed to develop, test and verify muon ionization cooling apparatus using the intense 400- MeV Fermilab Linac proton beam. The purpose of the facility was to test the basic techniques and components proposed for muon ionization cooling in a proton beam judged equivalent in impact to a muon beam for a Neutrino Factory or Muon Collider. Since the experimental scenarios developed for muon facilities required collection, capture, and cooling of large-emittance, high-intensity muon beams $\left(\sim 10^{13}\right.$ muons at a repetition rate of $\left.15 \mathrm{~Hz}\right)$ conclusive tests of the apparatus required full Linac beam, or $1.6 \times 10^{13} \mathrm{p}$ at $15 \mathrm{~Hz}$ [1]. General Linac beam parameters are listed in Table 1 for reference. However, based on installed shielding, but primarily due to line-of-sight large (4"-10") penetrations for cryogens and even larger gas vents, the integrated hourly rate in MTA currently is significantly less than the full $15 \mathrm{~Hz}$ Linac capability. Further, currently the primary beam is entirely deposited in the muon cooling apparatus and a beam stop installed in the experimental hall. Configuration control in terms of local shielding and active radiological controls have not been feasible given the large physical parameters of the muon apparatus (such as the SC solenoid and $\mathrm{RF}$ cavities) to improve intensity. In contrast, it is anticipated that $\mu \mathrm{SR}$ targets can be locally shielded to gain 2 orders of magnitude in passive shielding given that more than 6' is available radially around the target and will be discussed in this report. Transport of the primary beam either to a high-intensity beam absorber buried in the berm (downstream of the experimental hall) is required or, alternatively, to a new absorber installed in the experimental hall (closer to the production target). Both scenarios are investigated.

With the 2015 termination of the Muon Accelerator Program, and, specifically, the MICE cooling experiment, the users of the beamline are phasing out and the current purpose of the MTA experimental facility is ending with the 2017 Fermilab accelerator shutdown. The MTA is a powerful, world-class intensity proton facility and one exciting possibility with far-reaching applications would be conversion to a Muon Spin Resonance $(\mu \mathrm{SR})$ experimental facility. 
Table 1. Linac full capability beam parameters

\begin{tabular}{|l|l|l|}
\hline Parameter & Value & Unit \\
\hline Kinetic Energy & 401.5 & $\mathrm{MeV}$ \\
\hline Energy Spread & 1 & $\mathrm{MeV}$ \\
\hline RF Structure & 201.24 & $\mathrm{MHz}$ \\
\hline Bunch Length & 0.208 & $\mathrm{~ns}$ \\
\hline Pulse Length & 30 & $\mu \mathrm{s}$ \\
\hline Max Particles Per Bunch & 1.6 & $10^{9}$ \\
\hline Max Particles Per Pulse & 1.6 & 1013 \\
\hline Peak Current & 52 & $\mathrm{~mA}$ \\
\hline Mean Current & 38 & $\mu \mathrm{A}$ \\
\hline Max Beam Power & 15.7 & $\mathrm{~kW}$ \\
\hline Beam Emittance (99\%) & 8 & $\mathrm{~mm}-\mathrm{mrad}$ \\
\hline
\end{tabular}

\section{Muon Spin Resonance}

Muon Spin Resonance involves embedding polarized, positively charged muons in a sample material which allows the properties of the material to be measured and characterized at the microscopic atomic-structure level. The muons produced must be of sufficiently low energy that they stop within a few millimeters of material. Intense muon beams are currently produced first through bombardment of a low-Z production target with protons. The pions produced in nuclear reactions then decay "at rest" near the surface of the production target into low energy muons with polarization as high as $100 \%$ (a $\pi^{+}$at rest decays into a $4.120 \mathrm{MeV}$, or $29.792 \mathrm{MeV} / \mathrm{c}$, $\mu^{+}$and a neutrino). The polarized muons are transported via a low-energy beamline to the sample material. The spin of the muon couples to the local magnetic field of the material, making them sensitive probes of the magnetic environment. The $\mu^{+}$decays within a short time $\left(\tau_{\mu}=2.197 \mu \mathrm{s}\right)$ to a positron and two neutrinos, with the positron emitted preferentially in the direction of the initial $\mu^{+}$spin. Detection and measurements of the positrons reveals evolution of the $\mu^{+}$ polarization in the sample material, from which the properties of the target material can be determined.

The $\mu \mathrm{SR}$ techniques have found widespread application across diverse material-science fields, including atomic and molecular structure, solid state physics, superconductivity, and chemistry [see, for example, ref. 2]. At present, there are four operating accelerator $\mu \mathrm{SR}$ facilities in the world, with TRIUMF hosting the only North American center. At ISIS, PSI, and J-PARC the proton beams are optimized for production of neutron beams for neutron scattering experiments, not muon production. Muon production runs parasitically adapting to neutron production with strict limits on the degree to which the primary proton intensity available for the neutron program can be reduced. TRIUMF is the exception but its primary role is to service a wider nuclear community. The TRIUMF muon facility and operation would most resemble a future $\mu \mathrm{SR}$ facility at MTA. 


\section{Scenarios for $\mu$ SR at MTA}

There are two primary figures of merit in $\mu$ SR production, the target design and the facility intensity. Target considerations include the nuclear interaction length, $\lambda_{\text {nuc }}$, physical length, and its surface area. The second figure of merit is the yearly number of Protons On Target (POT) that can be delivered. As the MTA facility now stands, one present (option a) and two future scenarios (b and c) are explored; the present state of the facility, option a), is not considered competitive.

a) Current Facility: The current MTA experimental hall is limited to $5.3 \times 10^{5}$ POT/year $\left(60\right.$ pulses/hr) and in the upstream stub $5.3 \times 10^{6}$ POT/year $(600$ pulses/hour) for a $10 \%$ radiation interaction length target $[3,4]$ due to large penetrations for cryogenics, gas lines, and venting as can be seen in Figure 1. (Beam pulses can be taken at $15 \mathrm{~Hz}$ sequentially within an hour timeframe and then beam paused at the limit until the hour expirees.)

b) Re-purposed MTA: If penetrations are filled, the high-intensity beam absorber located at the exit of the hall in the berm can be used for high-intensity $15 \mathrm{~Hz}$ Linac operation. Recent Illinois, state-dictated groundwater contaminant levels limit POT/year to $2 \times 10^{19}[5]$. Local target shielding ( $\sim 6^{\prime}$ radially of steel and concrete) should permit 60,000 pulses/hour to be supported, or full Linac intensity. However, the phase-space acceptance of the buried bermpipe to the high-intensity beam absorber limits the target interaction length $\lambda_{\text {nuc }}$ to $5 \%$ and a light Be target, as derived in this report. This option represents an immediate, low cost approach to initiate a $\mu \mathrm{SR}$ facility in the near term within the operational constraints of the current civil construction with limited added shielding. Due to the target limitations imposed, option c) was added to allow a broader scope investigation into enhanced surface muon production and collection, especially in regard to the target and absorber design.

c) Conversion to a Target Hall: This option assumes the MTA experimental hall can be converted into a high-intensity target hall with a target pile followed closely by a high-intensity beam absorber (which must be similar in concept to the existing beam absorber for full intensity). The restrictive groundwater specifications will remain essentially identical since the beam height limits shielding that can be installed. An enhanced muon production target, one that would not be as limited by beam-blowup, would permit increased multiple scattering whether through higher $\mathrm{Z}$ or a longer target and thereby increase pion production. Surface muon production, however, is complex and there are limits to the transverse dimensions of the downstream absorber and to backscatter and radiation levels in the hall. Other issues such as air activation would need to be addressed and potentially structural ones such as the load capacity of the floor. This is a longer term, significant, and higher-cost modification to the facility and operation. 


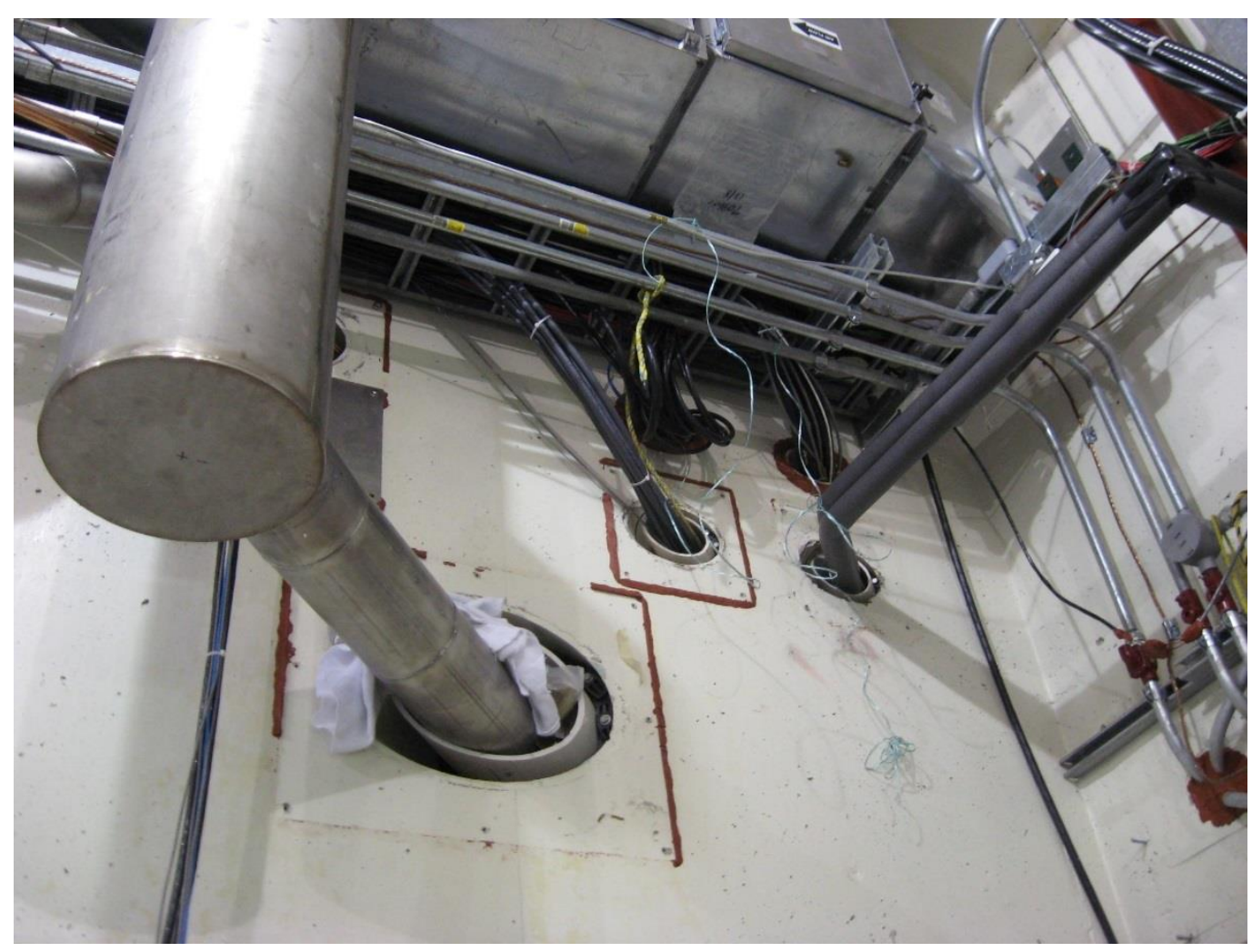

Figure 1. Large (up to 10") penetrations near ceiling of MTA experimental hall for cryogenics.

For comparison, TRIUMF accelerates $\sim 10^{15} \mathrm{p} / \mathrm{s}$ to $500 \mathrm{MeV}$, typically runs at 100-120 $\mu \mathrm{A}$ [6], and delivers, on average, $\sim 1.5 \times 10^{22} \mathrm{POT} /$ year to a muon production target. The PSI cyclotron operates at $590 \mathrm{MeV}$ with a proton current of $2200 \mu \mathrm{A}$ [7], thus potentially delivering a maximum of $4 \times 10^{23}$ POT per year on two graphite production targets. ISIS produces the only pulsed $\mu$ SR beam, delivering $2.5 \times 10^{13}$ protons at $50 \mathrm{~Hz}$ onto a graphite target [8]. Muon production, however, at ISIS is limited to a $4 \%$ production target due to their downstream neutron program. The pulsed Fermilab Linac delivers about a factor of 4 less protons than ISIS, mainly due to the repetition rate, but, as a dedicated muon facility, it could, in principle, support an enhanced production target. The PSI facility supports seven secondary derivative beamlines, at different viewing, or production angles exploiting the isotropic nature of surface muon production. At the MTA facility three such secondary muon beamlines could be implemented - the lines would probably require extension through the rollup door shield wall. Further, ISIS $\mu$ SR experimenters report that half an hour of irradiation can yield sufficient statistics for an experimental sample spectrum [9]. This translates to $\sim 2 \times 10^{18}$ POT, or about a couple of hours of full Linac intensity assuming an ISIS production target. The groundwater yearly limit, however, if not mitigated through increased shielding, would restrict the number of experiments that could occur at MTA/year to about 10. This would advocate for a new absorber design if the star density could be reduced - or radiation levels monitored which is possible within the hall.

This report first explores a dedicated muon facility primarily in terms of an optimized muon production target without regard to civil impact or costs and then discusses and derives what the 
present facility can support through a modest upgrade. This latter proposal provides a low-risk immediate path to a $\mu \mathrm{SR}$ facility. The three beamlines proposed could be supported in either scenario.

\section{Muon Production}

Specifications for a $\mu$ SR production target entail a) high yield of pions decaying to muons in the target, b) maximizing the rate of collected surface muons, and c) minimizing production of unwanted particles; i.e. electrons and positrons, neutrons, scattered protons, and gamma rays. A fraction of the pions produced have sufficiently low energy that they lose their entire momentum within the target and decay at rest producing a low, narrow-energy band of muons with a high degree of polarization in the direction of travel. Pions that decay in flight sufficiently close to the production target (to be collected), cloud muons, have a lower net polarization because of the large uncertainty in parent momentum and represent a background. Only muons formed close to the target surface, or surface muons, escape and these are positive because low-energy negative muons are captured by the target nuclei. A high stopping density of low-energy pions in the target is one goal of the target design to produce an intense secondary beam of surface muons of $10^{-8}-10^{-9} \mu+/ \mathrm{p}$.

Surface muons exhibit a momentum of 0-30 MeV/c, depending on the depth at which they were produced. Muon capture for a $\mu$ SR secondary beamline typically occurs in the momentum range 26-28 MeV/c (3-4 MeV kinetic energy, dp/p of $\pm 5 \%$ ) to allow collection, beam transport, and stable optics to the experimental target. At these low energies, there is minimal - a few percent - contamination from in-flight decay muons. Low-Z materials have been used for all $\mu \mathrm{SR}$ targets to date because they maintain a high rate of pion production while minimizing the contribution of multiple scattering to the outgoing primary proton beam. Optimizing production target design is a non-trivial undertaking, with the muon yield highly sensitive to the details of the target coupled to secondary muon beamline design. The following discussion illustrates sensitivity to the details of the target by summarizing findings from simulations performed for the ISIS facility.

Extensive GEANT4 simulations of muon production on the ISIS graphite production target have been published [10,11]. Although the calculations used ISIS as the reference, the conclusions and concepts for optimizing the production target design apply in general. The ISIS muon production target is a thin $7 \mathrm{~mm}$ graphite installed at a $45^{\circ}$ angle with respect to the proton beam and installed upstream of the neutron production facility. Muons are extracted at $90^{\circ}$ relative to the primary beam.

Simulations of muon yields as a function of incident proton energy from threshold to 3 $\mathrm{GeV}$ were studied for pions produced at $30 \mathrm{MeV} / \mathrm{c}$ or less for $10^{9} \mathrm{POT}$. The results are shown in Figure 2 which clearly show a peak yield around $500 \mathrm{MeV}$ as the optimal proton energy for surface muon production. (Total pion cross section and production increases with energy but surface and low energy muons that escape the target show this strong low-energy peak.) Also indicated is the relative yield that would correspond to the MTA energy of $400 \mathrm{MeV}$. This is $\sim 80-85 \%$ of the peak yield and is, encouragingly, equal to the ISIS $800-\mathrm{MeV}$ result. 


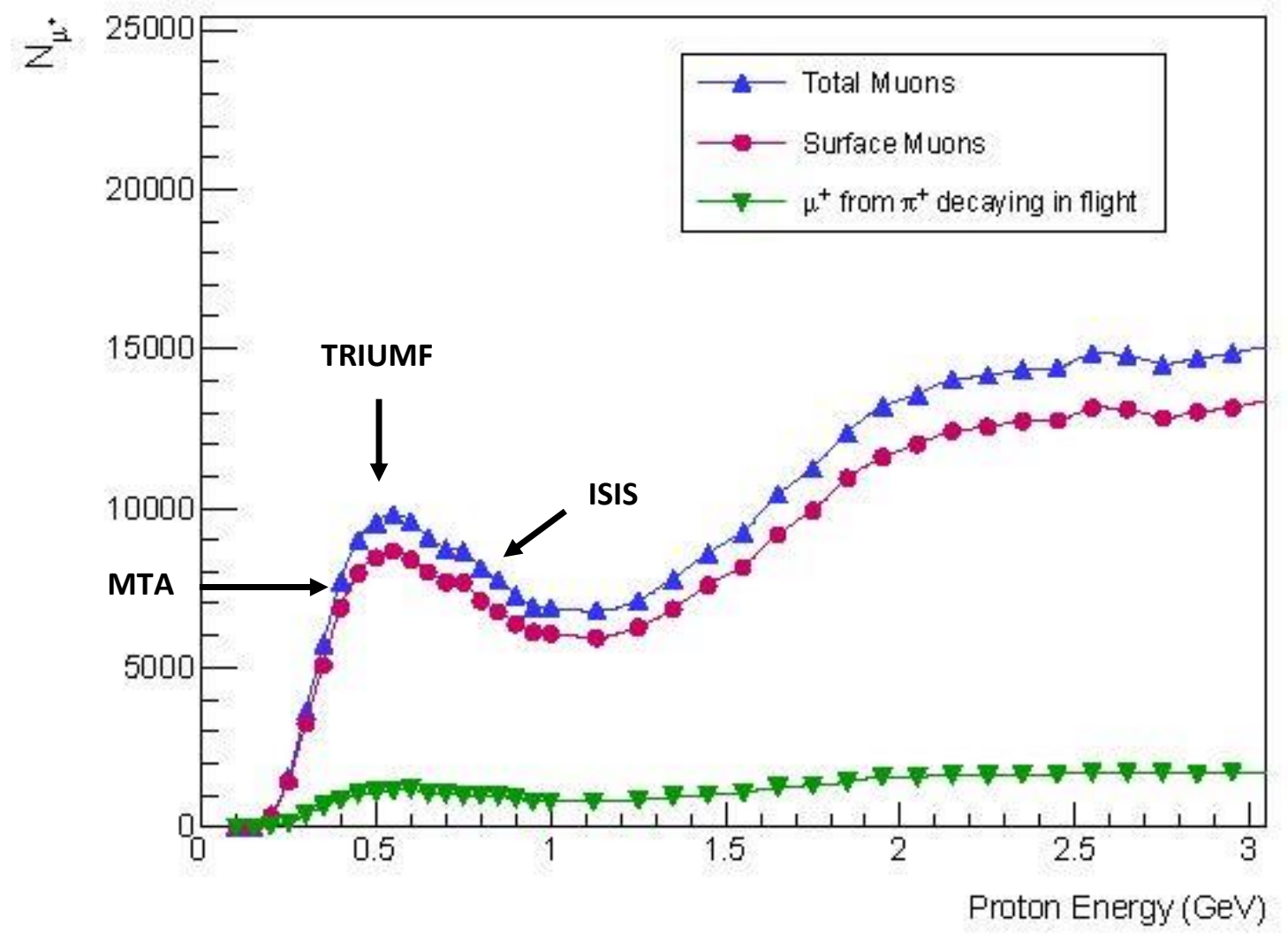

Figure 2. Variation of $\mu^{+}$yield with incident proton energy [10] for muons with momenta $\leq 30 \mathrm{MeV} / \mathrm{c}$; i.e. surface muons.

This is to be compared with the total pion production which strongly increases with energy: 


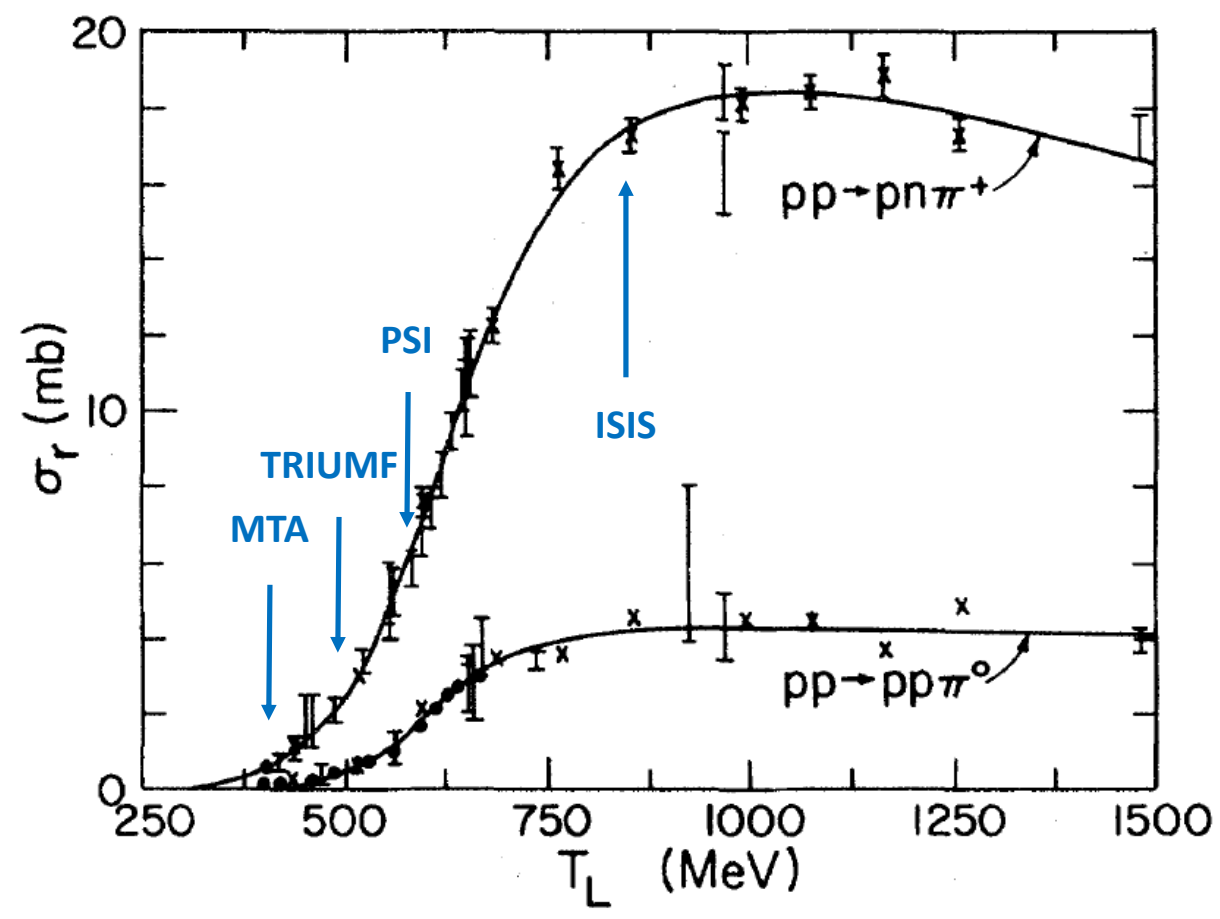

Figure 3. Total single pion production cross section dependence on proton energy [12].

The ISIS nominal beam current is $200 \mu \mathrm{A}$ at $800 \mathrm{MeV}\left(1.25 \times 10^{15} \mathrm{p} / \mathrm{s}\right)$. However, only a small fraction of this beam $\left(\approx 4 \%\right.$ or $\left.5 \times 10^{13} \mathrm{p} / \mathrm{s}\right)$ is available for $\mu$ SR studies. A proton transmission rate of $\sim 96 \%$ and low multiple scattering must be maintained to ensure that the neutron program is not significantly impacted. Such a constraint is not immediately applicable to a $\mu$ SR facility at MTA where with additional shielding the primary intensity could potentially reach $24 \times 10^{13} \mathrm{p} / \mathrm{s}$ at full Linac capability; equivalent to ISIS muon production for a $24 \% \lambda_{\text {nucl }}$ Be target, for example, which is one of the targets implemented at TRIUMF [8]. One limitation of the current MTA facility, as will be discussed in later sections, is low-loss transmission to the high-intensity absorber, unless a new absorber or civil modifications are made to the existing absorber. The next sections discuss relevant target considerations for muon production.

\section{Surface Muon Production}

Although pion production is forward/backward angle asymmetric, surface muon production is isotropic, as expected from a pion at rest, and not strongly dependent on incident primary proton energy (threshold is $280 \mathrm{MeV}$ ). To demonstrate this asymmetry, the momentum distributions of pions and muons were calculated as functions of proton energies for $\pi^{+}$and $\mu^{+}$ inside of $20^{\circ}$ relative to the forward and backward directions [10]. The pion distribution is strongly forward biased (which increases with beam energy). By contrast, the muon momentum shows no backward-forward asymmetry (suggesting that collection of backward muons is preferable for transporting a clean $\mu^{+}$beam). Figure 4 shows simulation results at a representative energy of 500 $\mathrm{MeV}$ for the incident protons [10]. 
Isotropic production is very powerful in that it allows the prospect of multiple secondary muon lines viewing the same production target at different angles, as is implemented at PSI where 7 beamlines access two production targets. (ISIS has two $90^{\circ}$ beamlines but on opposite sides of the target.) However, the forward pion asymmetry implies that a forward angle viewing within $20^{\circ}$ would likely intercept tails of the primary proton beam even for a $4 \%$ graphite target. A balance must be achieved between multiple beamlines and multiple scattering, the latter a function of target length and atomic number. For the ISIS $45^{\circ}, 7 \mathrm{~mm}$ graphite target, a beamline could not be implemented near the defining $20^{\circ}\left(\theta_{\mathrm{rms}}\right.$ is only $2.23 \mathrm{mr}$ ). For longer, or heavier, targets, the effects of multiple scattering increase and must be considered in the orientation and acceptance of multiple secondary beamlines. The next section discusses studies to optimize production and collection (escape) of surface muons into a secondary beamline.
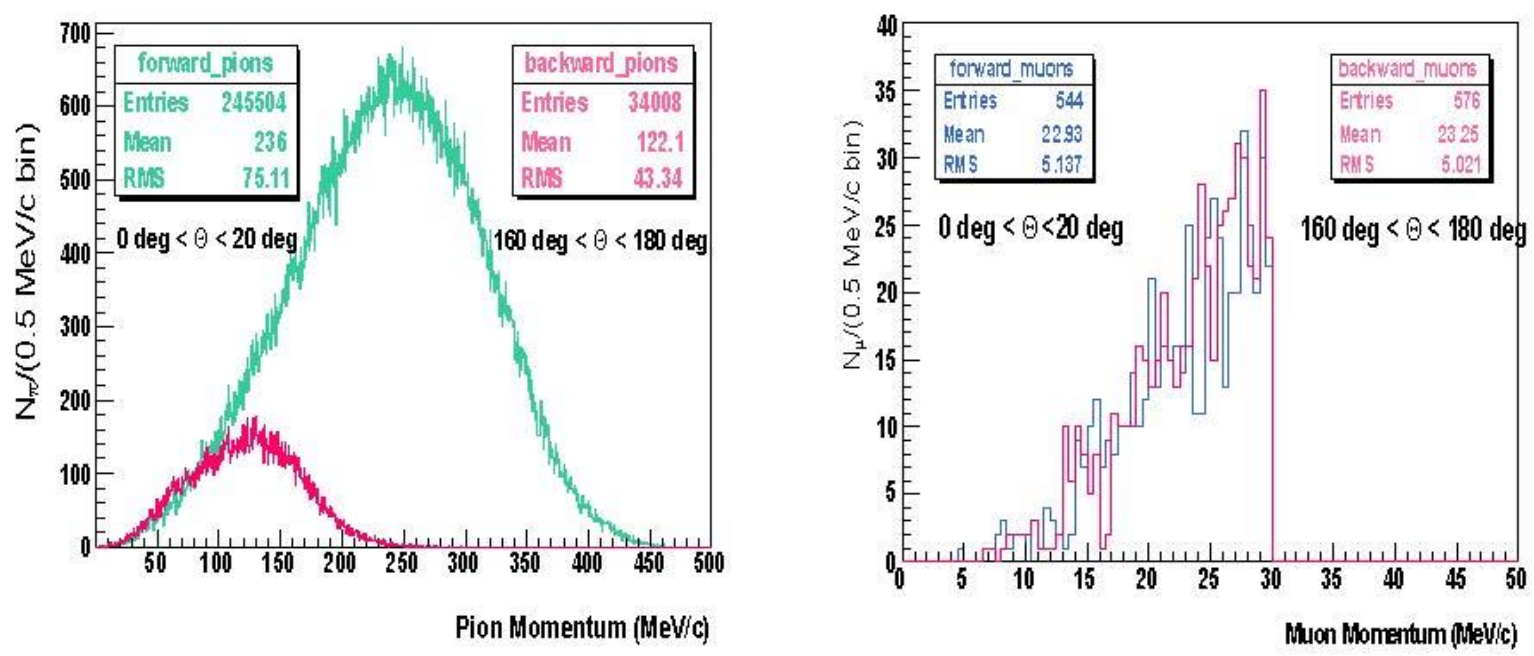

Figure 4. Pion momentum distribution for $500 \mathrm{MeV}$ protons incident on graphite (left) and corresponding $\mu^{+}$distribution (right) inside forward (green and blue) and backward (red and purple) within $20^{\circ}[10]$.

\section{Target Design}

Low-energy, surface muon yield is highly sensitive to the geometric details of target design and relative orientation to collection. A surface phenomenon necessarily involves maximizing surface area, minimizing secondary pion absorption, and, importantly, promoting the diffusion of ultra-low energy muons to the surface without significant energy loss. Only muons at the highend of the production spectrum are collected by the secondary beamline ( $27 \mathrm{MeV} / \mathrm{c} \pm 5 \%$ which is a large momentum acceptance for a beamline). Optimizing the production target for surface muon production emphasizes increasing surface area to volume; producing more muons near the surface implies thin targets which also reduces secondary pion absorption and energy loss in transit. Increasing the target angle away from the normal is a highly effective way to increase the number of nuclear interaction lengths without consequentially impacting the surface to volume 
ratio and muon mean path to the surface. However, the collection, acceptance, and transmission of the secondary line must be factored into the optimization as it establishes limits on target optimization parameters - associating target dimensions with secondary beamline acceptance, for example. $\mathrm{Z}$ dependence and target length are not simple contributions as they are in pion production or in conventional predominantly forward secondary production lines. Higher $\mathrm{Z}$ and shorter target lengths could, in principle, allow thinner targets and increased proton interactions for the same acceptance solid angle, but increased large-angle primary proton contribution to backgrounds must be assessed along with primary beam containment.

In the ISIS study, to maximize surface, the researchers simulated splitting the graphite target into slices along the beam path while maintaining the nominal proton transmission efficiency of $96 \%$ and studying the impact of number of slices and separation. Target angle was also studied to enhance the effective target length and collection efficiency. Splitting the slab into two slices doubled the surface area without increasing the total thickness - more pions decay at rest near the surface allowing more muons to escape the target. Roughly, as the slice separation increased more surface muons escaped from the target region and were collected. Collection saturated at approximately 50-60 mm separation for a $45^{\circ}$ target angle as shown in Figure 5 (which increases the total interaction length by $40 \%$ from the normal). As mentioned, practical assumptions as to the acceptance of the secondary muon beamline must be incorporated into the target optimization in order to properly analyze and compare target concepts. The ISIS beamline collects surface muons across a solid angle of 0.071 steradians through an $8 \mathrm{~cm} \mathrm{Al}$ window located $15 \mathrm{~cm}$ from the center of the target. This window aperture also reflects quadrupole apertures in the secondary beamline. If this secondary line acceptance is folded in, the number of slices and slice separation reach optimal values at 3 slices and $20 \mathrm{~mm}$ separation as shown in Figure 6. Dependence on target angle was also studied but optimization ultimately depends on the beamline(s) orientation. For the ISIS study, the $45^{\circ}$ target angle was optimal but there the beamlines are normal to the primary beam direction. Within the existing ISIS extraction and transport configuration these simple modifications to the ISIS target were predicted to double the detected surface muon yield (from 7 $\mathrm{x} 10^{-9} \mathrm{\mu}^{+} / \mathrm{p}$ to $15.5 \times 10^{-9} \mu^{+} / \mathrm{p}$ ). If the primary proton transmission is not constrained, heavier targets can be considered. 


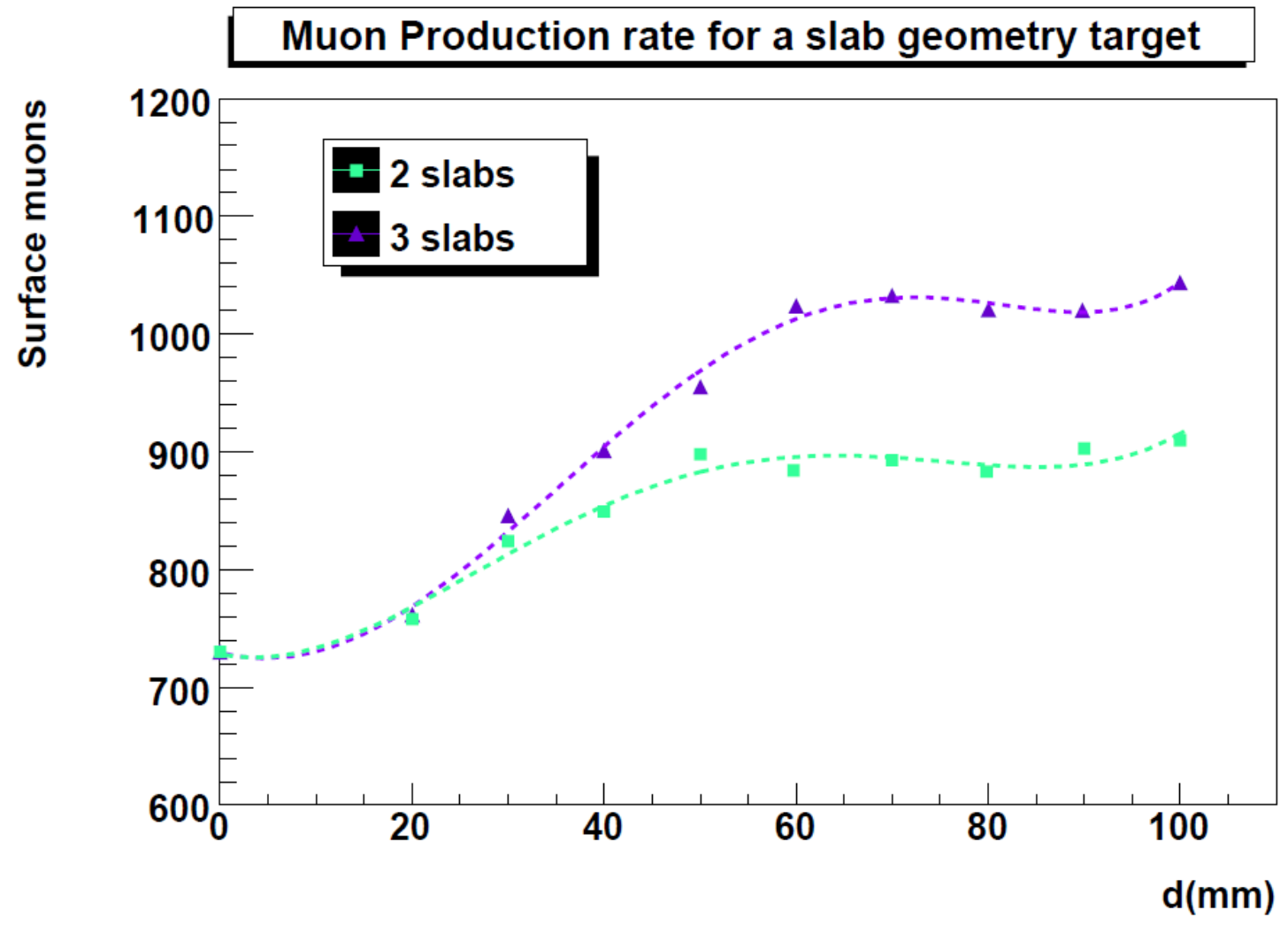

Figure 5. Variation of the number of surface muons with distance between target slices [13] 


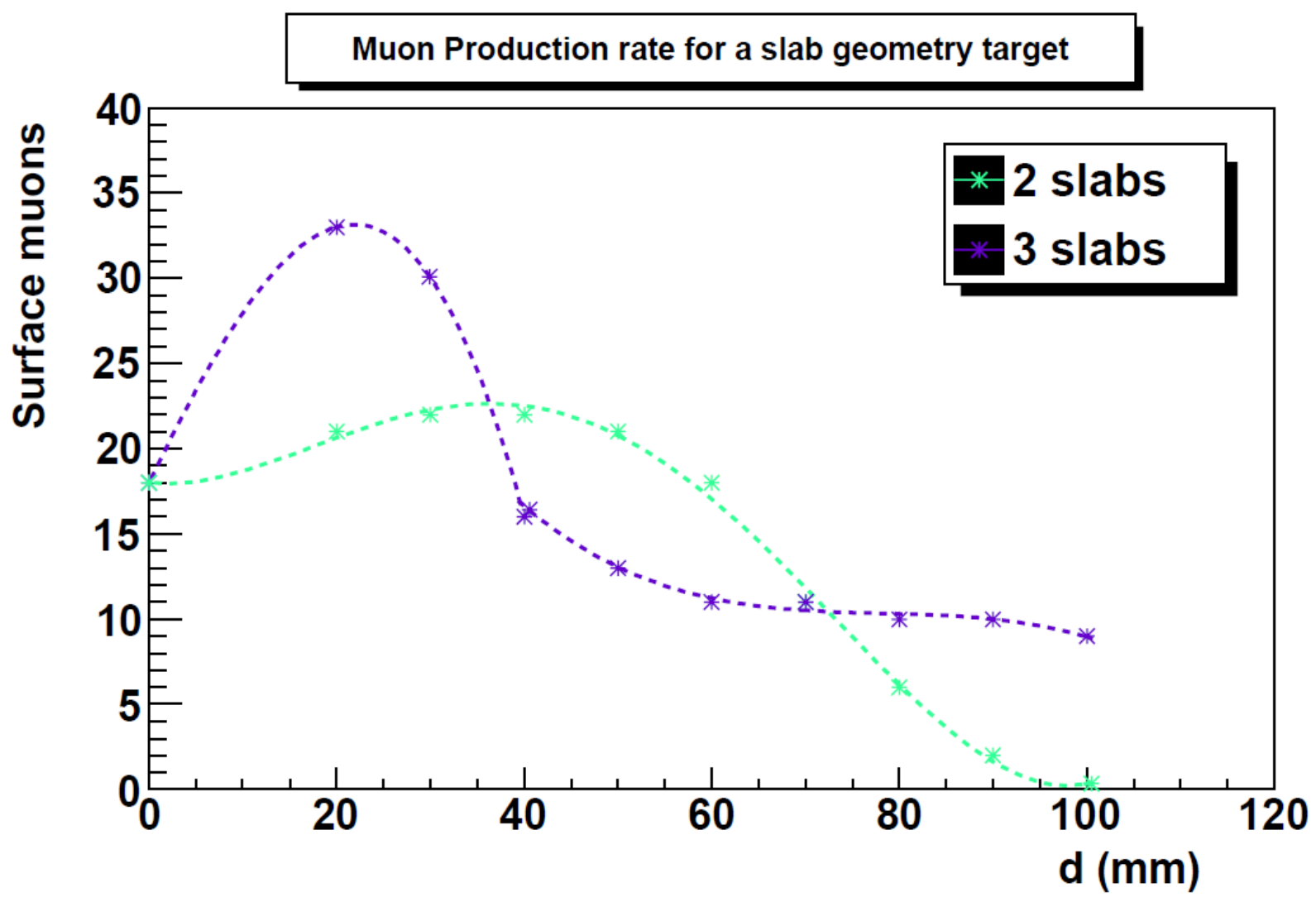

Figure 6. The number of surface muons collected at the ISIS entrance window to the muon channel beamline with distance showing coupling of target design to muon beamline design [13].

\section{Target Material}

The target material and design, especially in terms of target cooling must be considered simultaneously. At PSI a $1 \mathrm{~Hz}$ graphite target wheel with radiative cooling is used [7] but at ISIS active edge cooling is used [8]. Required thermal and mechanical properties are discussed followed by a discussion of pion production and $\mathrm{Z}$ dependence.

\section{Thermal and mechanical properties}

Like ISIS, the Fermilab target must survive the extremely high peak energy deposition of an intense pulsed proton beam ( $50 \mathrm{~mA}$, or $20 \mathrm{MW}$, delivered in $30 \mu \mathrm{sec})$. The target must not only survive (and dissipate) this instantaneous energy deposition, but also the thermal shock and the longer-term radiation damage. The vacuum conditions exacerbate the temperature so only target candidates with high melting points or the ability to rapidly conduct heat away are considered. This limits the target materials effectively to $\mathrm{Be}$, graphite, $\mathrm{Ti}, \mathrm{Cu}, \mathrm{Ni}$, and $\mathrm{W}$ whose melting points fall in the range from 1287 to $4000^{\circ} \mathrm{C}$. Only $\mathrm{Cu}$ is lower at $1085^{\circ} \mathrm{C}$, but is attractive for its superior high conductivity and, therefore, conductive-based cooling properties. (It was found to be the best material for the core of the high-intensity MTA absorber [14,15]. Note that in Figure 7, steel melts 
in the Fermilab full-intensity beam.) Low expansion coefficients are also desirable (Be). Ni is a choice for its high melting point $\left(1760^{\circ} \mathrm{C}\right)$ and stress resistance.

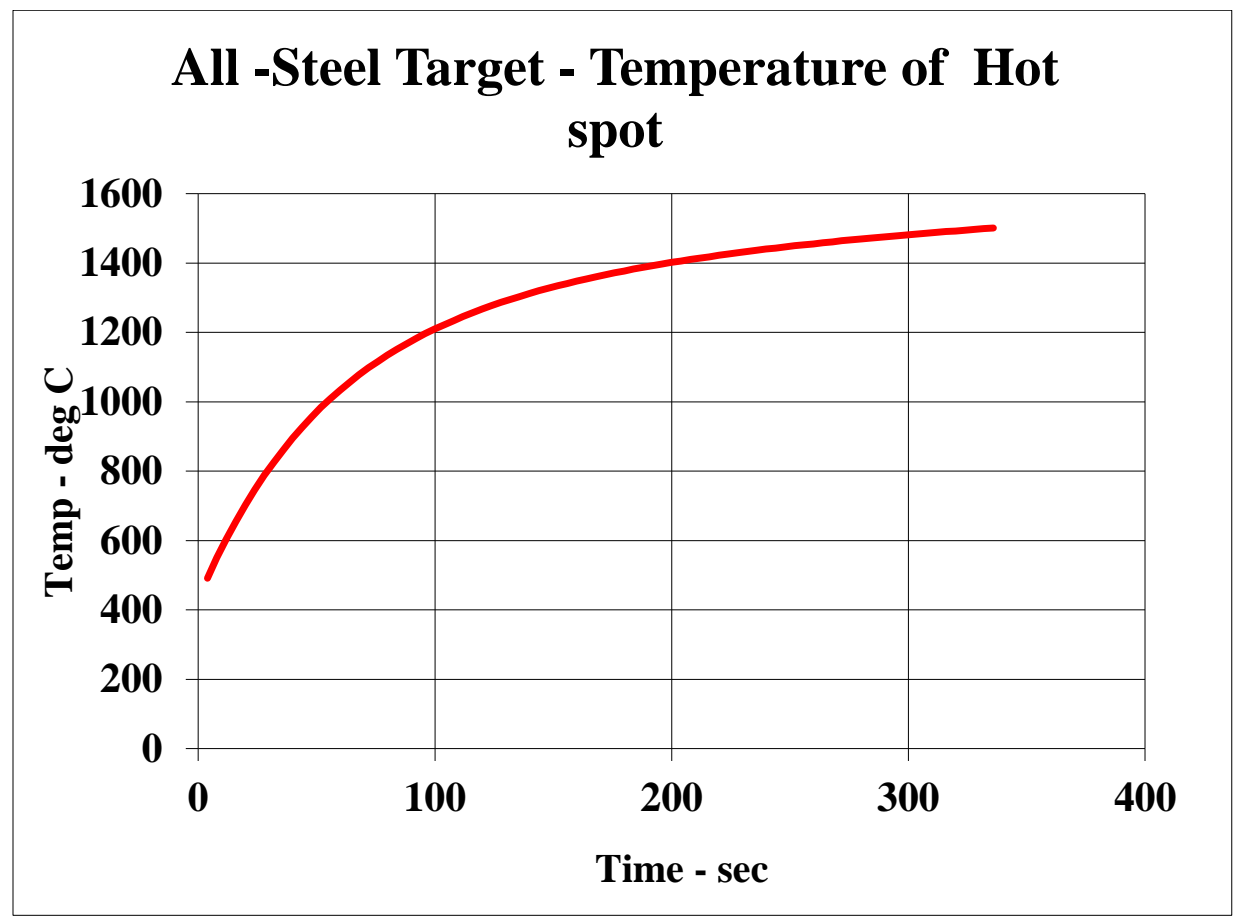

Figure 7. An all-steel dump operated at full Linac intensity [16,17]. Steel melts after 3 minutes of beam at $1370^{\circ} \mathrm{C}$ for full beam absorption

\section{Z-dependence}

Overall pion production and muon yield depends on choice of target material as well as geometry. To date, low-Z materials have been chosen at all $\mu \mathrm{SR}$ facilities in order to maximize pion production and minimize both the rate of absorption of secondary pions and multiple scattering of the incident proton beam by the production target (multiple scattering to nuclear interaction length is lowest for Be). Muon yield simulated for three different target materials: beryllium, graphite, and nickel as a function of target thickness is shown in Figure 8. For a dedicated $\mu$ SR facility, nickel would produce a much higher muon yield than both graphite and beryllium for targets of equal length. However, when analyzed in terms of the yield per nuclear interaction length, interestingly, $\mathrm{Ni}$ and graphite are equivalent, and both are more productive than Be as shown in Figure 9. This analysis needs to be repeated for $400 \mathrm{MeV}$ protons.

In a dedicated muon facility where the primary proton transmission can be relaxed to a less critical tolerance, for the same thickness (maintaining the advantage of surface to volume ratio and beamline acceptance) and the same target geometry, a nickel target, for example, would produce a substantially higher muon yield than graphite and beryllium due to decreased nuclear interaction length. [Pion production on nuclei is highly complex, however, and not well understood: Phenomenological parameterizations of $\pi^{+}$production follow a $Z^{1 / 3}$ dependence whereas $\pi^{-}$ 
production varies with the number of neutrons as $\mathrm{N}^{2 / 3}$ (see, for example, ref. 18)]. Higher-Z target materials up to tungsten can be investigated and compared to the graphite target study using equations derived below. The caveat is that the radiation length is decreasing rapidly so the size of the downstream primary beam is increasing more rapidly than pion production with the size of the absorber core growing in proportion to $\theta_{\mathrm{rms}}$. A comparative analysis of the two competing specifications is performed here. Contamination levels from the scattered primary proton beam into the secondary line, however, would have to be studied in detail as a function of $\theta_{\mathrm{rms}}$, beamline orientation, acceptance and optics before a target and beamline design can be confirmed.

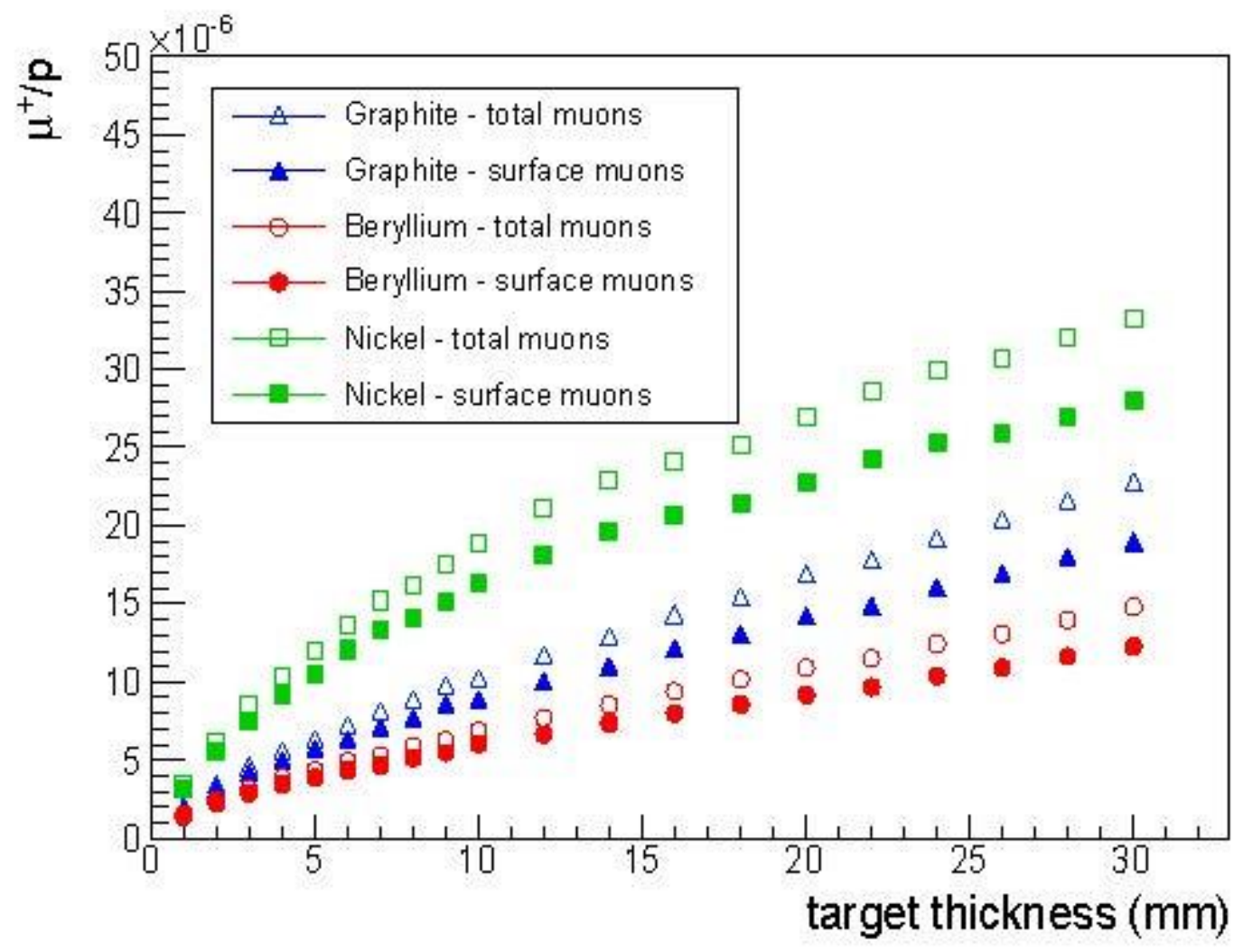

Figure 8. Comparison of muon yield for $800 \mathrm{MeV}$ incident protons on 3 different materials a function of target thickness [10]. 


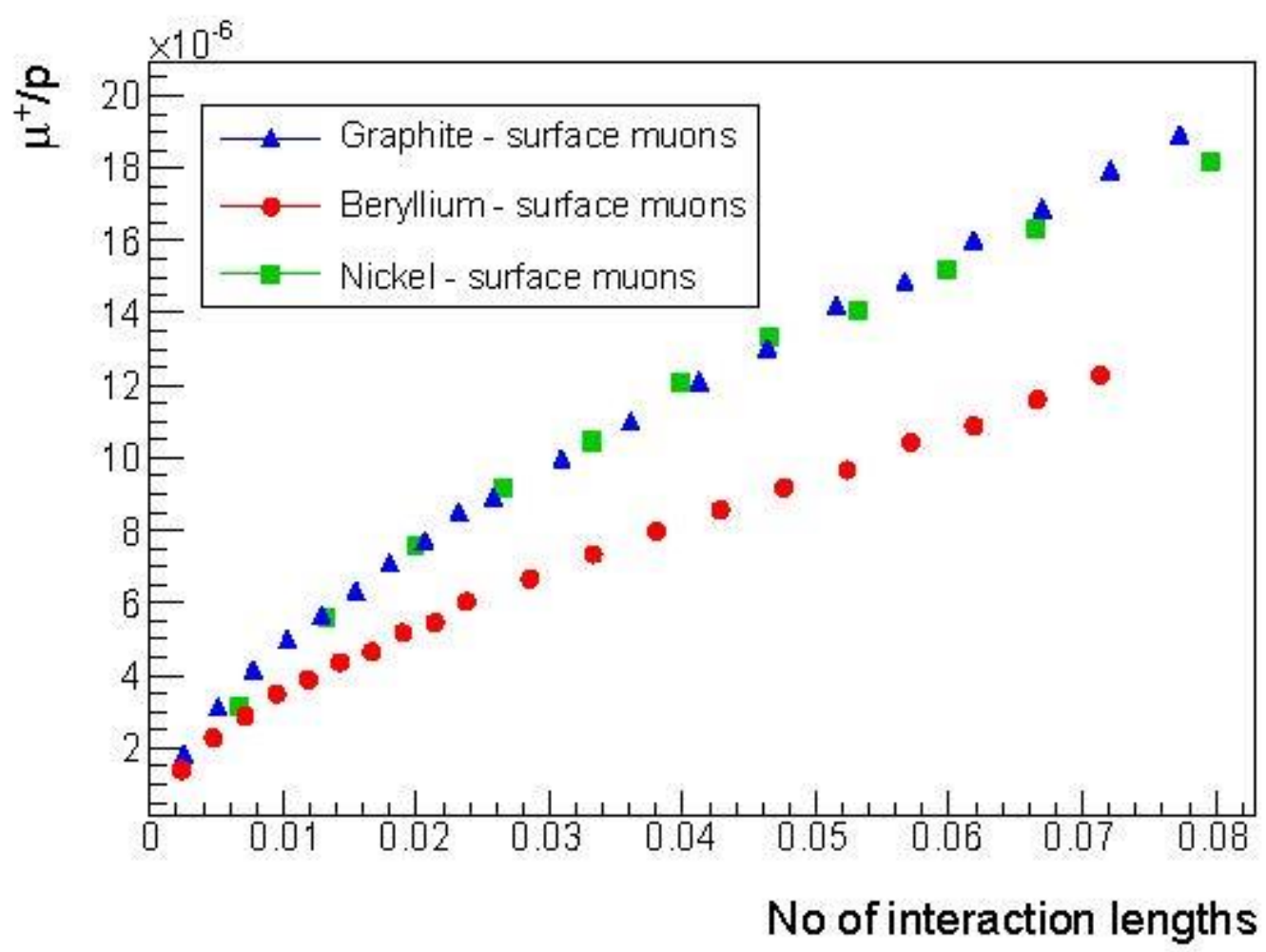

Figure 9. Comparison of muon yield for $800 \mathrm{MeV}$ incident protons on 3 different materials as a function of interaction length [10].

In order to design a beam capture and/or transport system, limits must be set on the properties of the outgoing primary beam cone since the parameters of this cone are dominated by multiple scattering in the production target. Specifying the gross properties of the downstream primary beam can be accomplished using the multiple scattering angle as defined by the conventional equation [for example, ref. 19]:

$$
\theta_{\text {rms }}=\frac{13.6 \mathrm{MeV}}{p v} \sqrt{\frac{l_{\text {tgt }}}{L_{\text {rad }}}}\left[1+0.038 \ln \left(\frac{l_{\text {tgt }}}{L_{\text {rad }}}\right)\right]
$$

If the ratio $\mathrm{l}_{\mathrm{tgt}} / \lambda_{\mathrm{rad}}$ is fixed, then $\theta_{\mathrm{rms}}$, or the opening cone angle, is determined independent of target material.

$$
f=\frac{l_{\text {tgt }}}{\lambda_{\text {rad }}} \text { where } f \text { is a constant; }
$$

Assuming that pion production in a target scales to a good approximation with the number of $\lambda_{\text {nuc, }}$ of the target starting at graphite as Figure 9 implies (lighter elements appear to have a reduced production value) then relative pion production can be estimated in terms of $f$ and the ratio of radiation interaction length to nuclear interaction length.

$$
l_{\text {tgt }}=f \lambda_{\text {rad }}=k \lambda_{\text {nuc }} ; \quad \therefore k=f \frac{\lambda_{\text {rad }}}{\lambda_{\text {nuc }}}
$$


Then to estimate relative pion production as a function of target material, one can normalize to carbon.

$$
\left.\frac{k}{k\left({ }^{12} C\right)}=\frac{\left(\lambda_{\text {rad }} / \lambda_{\text {nuc }}\right)}{\left(\lambda_{\text {rad }}\left({ }^{12} C\right) / \lambda_{\text {nuc }}\left({ }^{12} C\right)\right.}\right)=2.0\left(\lambda_{\text {rad }} / \lambda_{\text {nuc }}\right)
$$

Since we know $\lambda_{\text {rad }}$ and $\lambda_{\text {nuc }}$ for ${ }^{12} \mathrm{C}$, a table of the pion production rates normalized to carbon can be generated independent of the value of multiple scattering assumed for the primary proton beam. For a fixed $\theta_{\mathrm{rms}}$, then, these results are independent of target length. The pion rate can also be compared against a fixed target length where the $\mathrm{Z}$ dependence dominates, but the scattered primary beam size grows much faster- rapidly reaching physical design limits depending on the facility and shielding accommodation (spallation source shielding design cannot be applied). There is little dependence of the relative absorber dimensions with target length for $1 \mathrm{~cm}$ to $8 \mathrm{~cm}$ which are reasonable choices for a $400-\mathrm{MeV}$ proton beam based on equivalent beamline apertures.

Table 2. Relative Muon Production normalized to ${ }^{12} \mathrm{C}$ for the same outgoing primary beam properties. Note that Be does not scale proportionally to the relative production equation and muon yield is assumed to be about a factor of 2 lower per Figure 9.

\begin{tabular}{|c|c|c|c|c|c|}
\hline $\begin{array}{c}\text { Target } \\
\text { Nuclei }\end{array}$ & $\begin{array}{c}\lambda_{\text {rad }} \\
(\mathrm{cm})\end{array}$ & $\begin{array}{c}\lambda_{\text {nuc }} \\
(\mathrm{cm})\end{array}$ & $\begin{array}{c}\theta_{\text {rms }} \text { constant } \\
\text { Relative } \\
\pi \text { production }\end{array}$ & $\begin{array}{c}\text { ltgt constant } \\
\text { Relative } \\
\pi \text { production }\end{array}$ & $\begin{array}{c}\text { Relative size of } \\
\text { absorber } \\
\text { ltgt }=1 \mathbf{c m} / \mathbf{8 c m}\end{array}$ \\
\hline${ }_{4} \mathrm{Be}$ & 35.28 & 42.10 & $\sim 0.54$ & $\sim 0.46$ & $0.72 / 0.72$ \\
\hline${ }_{6} \mathbf{C}$ & 19.32 & 38.83 & 1.00 & 1.00 & $1.00 / 1.00$ \\
\hline${ }_{22} \mathbf{T i}$ & 3.56 & 27.80 & 0.25 & 1.40 & $2.50 / 2.48$ \\
\hline${ }_{28} \mathbf{N i}$ & 1.424 & 15.06 & 0.19 & 2.58 & $4.09 / 4.06$ \\
\hline${ }_{29} \mathrm{Cu}$ & 1.436 & 15.32 & 0.19 & 2.54 & $4.08 / 4.05$ \\
\hline $74 \mathbf{W}$ & 0.3504 & 9.946 & 0.07 & 3.90 & $8.71 / 8.60$ \\
\hline
\end{tabular}

Column 3 in Table 2 reflects the fact for a fixed $\theta_{\text {rms }}$ the ratio of $\lambda_{\text {rad }}$ to $\lambda_{\text {nuc }}$ is decreasing dramatically with atomic weight. The dependence of $\lambda_{\text {rad }}$ on atomic weight is given by [20]:

$$
\lambda_{\text {rad }} \approx \frac{716.4 A}{Z(Z+1) \ln \left(\frac{287}{\sqrt{Z}}\right)} \mathrm{g} / \mathrm{cm}^{2}
$$

The nuclear interaction length is a much slower function of atomic weight [20]:

$$
\lambda_{\text {nuc }} \approx 35 \mathrm{~A}^{1 / 3} \mathrm{~g} / \mathrm{cm}^{2}
$$

Although qualitative, this approach provides a reasonable measure of the impact of target material beginning at graphite especially when it is noted that the $\mathrm{dE} / \mathrm{dx}$ of pions and muons is similar and their contributions approximately cancel [7]. The interpretation of these results is that 
for the secondary beam, the target insertion length is essentially fixed as determined by the beamline acceptance and muon yield always increases with the $\mathrm{Z}$, but for a downstream absorber the design is determined by the scattered primary beam and peak muon yield is maximized with a graphite target. These are conflicting optimizations, but the rapid change in $\lambda_{\text {rad }}$ dominates to the extent that practical target designs have used graphite or beryllium and remain the material of choice even for dedicated muon facilities. For an absorber in the MTA hall, titanium might be an acceptable choice but provides only $40 \%$ more muons at a cost of more than a factor of two in the downstream primary beam size and related absorber core dimensions - but such an absorber could be located in the hall close to the production target to offset increased size - although proximity is limited by backscattered radiation.

In summary, limits on the downstream primary proton beam must be established in order to design a feasible primary beam absorber and keep background levels in secondary lines to acceptable levels - especially for such low-energy, low-rate secondary lines. (Contamination from the primary beam and other production products are a serious design consideration.) If only the solid angle acceptance of the secondary line is considered, then replacing the target with the heaviest nucleus possible produces the highest muon yield approximately proportional to $Z^{1 / 3}$. [18]. Meanwhile the size of the primary beam and absorber is increasing much more rapidly than pion rate is. Still, if the acceptance of the line is defined and limits placed on the downstream primary beam, the target $\mathrm{Z}$ dependence can be revisited. The tradeoff is between secondary beamline acceptance and a feasible downstream absorber.

These results confirm the target research of $\mu$ SR facilities, even dedicated ones, which exclusively use the lighter $\mathrm{Z}$ targets, beryllium and graphite (recently boron carbide has been proposed [7]). Spallation targets have been studied and rejected [7]. Of all the facilities, TRIUMF sustains the largest multiple scattering of the incident proton beam with a $24 \% \lambda_{\text {nuc }}$ and a $28 \% \lambda_{\text {rad }}$

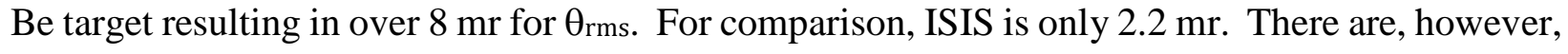
practical limits to the extent of the target which can be used and still effectively collect surface muons, the reason being that these beamlines are not forward as in normal secondary production lines but are at large viewing angles with respect to the direction of the incident proton beam due to the isotropic nature of surface muon production combined with the requirement for low backgrounds. These lines are generally at large angles (ISIS is $90^{\circ}$ and on either side of the production target). A practical secondary beamline acceptance is one limiting factor on the extent of the production target for this case and is discussed next. Independently, the production target length is limited by multiple scattering in terms of leakage of the primary beam and other production products into the secondary line.

\section{Secondary Muon Beamline}

Practical assumptions concerning the secondary beamline acceptance and orientation must be folded into the evaluation of target details in order to make even a preliminary recommendation for the target thickness and configuration for a feasible $\mu$ SR implementation at MTA. The ISIS conditions are most similar to present $400-\mathrm{MeV}$ apertures and available components for a secondary beamline; i.e. the $8 \mathrm{~cm}$ diameter $\mathrm{Al}$ window and downstream apertures are comparable to the $8.3 \mathrm{~cm}(3.25 ")$ apertures characteristic of MTA. The window is located $15 \mathrm{~cm}$ from the 
center of the target subtending $\Omega=0.071 \pi$. The ISIS beamline collects surface muons from \pm 30 and $\pm 5 \mathrm{~mm}$, and within 35 and $180 \mathrm{mr}$, horizontally and vertically, respectively, when projected back to the target. The acceptance of the ISIS secondary muon beam line is a geometric emittance of about $1000 \pi \mathrm{mm}-\mathrm{mr}$ and a dp/p of $\sim \pm 5 \%$ relative to a central momentum of $26.5 \mathrm{MeV} / \mathrm{c}$. In the single target simulation, a $7 \mathrm{~mm}$ thickness and a $45^{\circ}$ angle gave approximately $7 \times 10^{-9}$ surface $\mu^{+} / p$ transported by the secondary beamline to the experiment.

What is exciting about the MTA target is that the two slice approach can be extended to a thicker sample for two slices or three slices without a transmission constraint. The 2-slice geometry, for now, is assumed along with a $35-45^{\circ}$ target orientation which has been shown to increase muon yield without compromising collection from both sides of the target. The projected increase in muon collection is a factor of 2 based on the extensive ISIS studies. For an $8 \mathrm{~cm}$ target insertion length, roughly the acceptance limit of an $8 \mathrm{~cm}$ aperture normal orientation beamline, two thicker, $1-\mathrm{cm}$ slices can be installed with a $2 \mathrm{~cm}$ separation and $2 \mathrm{~cm}$ upstream and downstream for surface collection and, assuming a $45^{\circ}$ orientation angle, gives a total $2.8 \mathrm{~cm}$ target length along the beam direction. This configuration yields a factor of 2 from two slices and an additional factor of 2.8 from increased target thickness. The net gain in muon yield potentially exceeds that of an ISIS beamline.

In summary, target and muon beamline designs are complex, inter-dependent, and must be designed together. In the studies cited optimization of muon yield was constrained in the simulations by modeling of the existing extraction and muon transport hardware configurations. Due to the potentially large enhancements of yield attainable, investigators emphasized that even higher yields could be achieved if detailed target design preceded and were subsequently matched to extraction, collection, and transport considerations.

\section{Target Conclusions}

The conclusions as to target material can be understood simply. If graphite, for example, is replaced by nickel, clearly the muon yield will increase but so also will the outgoing primary beam cone. However, if a nickel target is replaced with an equivalent total number of $\lambda_{\text {nuc }}$ carbon target, then the pion production and muon yields will be as much as a factor of 4 higher for the same downstream primary beam properties (Note that the graphite target will be $\sim 2.6 \mathrm{x}$ longer). However, the length of the target insertion is coupled to and limited by the design of the secondary line.

A titanium target, for example, would increase the muon yield by about $40 \%$ at a cost of a much larger, $\sim 2.5 \mathrm{x}$, primary beam absorber core. By comparison, the target geometry is more effective than $\mathrm{Z}$ dependence, increasing the muon yield by about a factor of 2 without a corresponding increase in the absorber cross section. A combination of geometry and $\mathrm{Z}$ may be optimal but requires detailed production, collection and transport modeling.

What can be determined is the present capability of the MTA in comparison with ISIS. The Fermilab Linac is a factor of 4 lower in proton intensity than ISIS. However, removal of the $96 \%$ transmission limit combined with target improvements enable a secondary beamline with a 
potentially comparable and possibly higher rate of secondary muons on the sample material over ISIS performance. The net $2.8 \mathrm{~cm}$ long target proposed represents a $7.2 \% \lambda_{\text {nuc }}$ and a $14.5 \% \lambda_{\text {rad. }}$ As will be shown in a detailed discussion of current MTA capability, any value above $5 \%$ for $\lambda_{\text {nuc }}$ and this assumes beryllium, not graphite, requires a new implementation of a high-intensity absorber, most likely installed in the experimental hall. Still, the proposed target can be accommodated by a hall absorber design similar to the current high-intensity absorber. If target geometry optimization is also applied, the projected muon rate exceeds ISIS capability.

\section{MTA Overview}

The MTA experimental facility is supported by a primary beamline that extracts, transports, and delivers $400-\mathrm{MeV} \mathrm{H}^{-}$beam directly from the Linac through a 12' shield wall and into a "stub" which opens into a 40' long, 20' wide hall. The section of beamline upstream of the shield wall is housed in an enclosure contiguous with the Linac and is not accessible when the Linac accelerator is operating. Any access to the Linac side of the beamline is disruptive to Linac operations and generally is only allowed during an "opportunistic" or scheduled Linac shutdown. Therefore, the upstream beamline section is not operationally appropriate for a production target - despite the increased Linac berm shielding of 19' as required for full-current Linac operation (maintains lowoccupancy radiation levels outside of the shielding). The first experimentally accessible area downstream of the wall is a section of the beamline housed in a 30' stub that opens into the experimental hall, a civil remnant that remains from the 400-Mev upgrade to the Linac. The 12' shield wall that separates the upstream section of the beamline from the downstream beamline stub and experimental hall is complicated by the RF waveguides embedded within the shield wall, although in principle this could be disassembled through an existing hatch, but at a high cost. (If additional Linac signals are required or power cabling this may have to occur, but requires a crane with $\sim 100$ ' boom.) In the present enclosure configuration, the shield wall allows personnel access to the beamline stub and experimental hall during Linac operations which is ideal from an experimental user standpoint.

The layout of the beamline from the Linac through the shield wall to the high intensity dump is shown in Figure 10 [3,4]. The MTA currently supports two modes of beam operation: beam delivery to an upstream emittance beam absorber located at the end of the stub and used for performing detailed measurements of Linac beam properties [21], and; beam delivery to the MTA experiments. The second mode can either utilize a collimator and local absorber assembly that can be re-positioned as needed with respect to the experimental apparatus. Another variation in the experimental mode of operation allows for beam downstream of a strictly-defined experiment to be delivered to the high-intensity beam absorber buried in berm downstream of the hall. This latter mode has yet to be implemented and appears to require downstream magnetic components to confine and direct beam to the high-intensity absorber. Present limits for the two modes of operation are: 600 pulses/hour or $9.6 \times 10^{15}$ protons/hour for beam measurements and 60 
pulses/hour or $9.6 \times 10^{14}$ protons/hour to MTA experiments [16,22,23] ${ }^{1}$. The two modes are referred to as the 'Emittance' mode and 'Experiment' mode, respectively. Beam intensity parameters for the two operational modes are as follows.
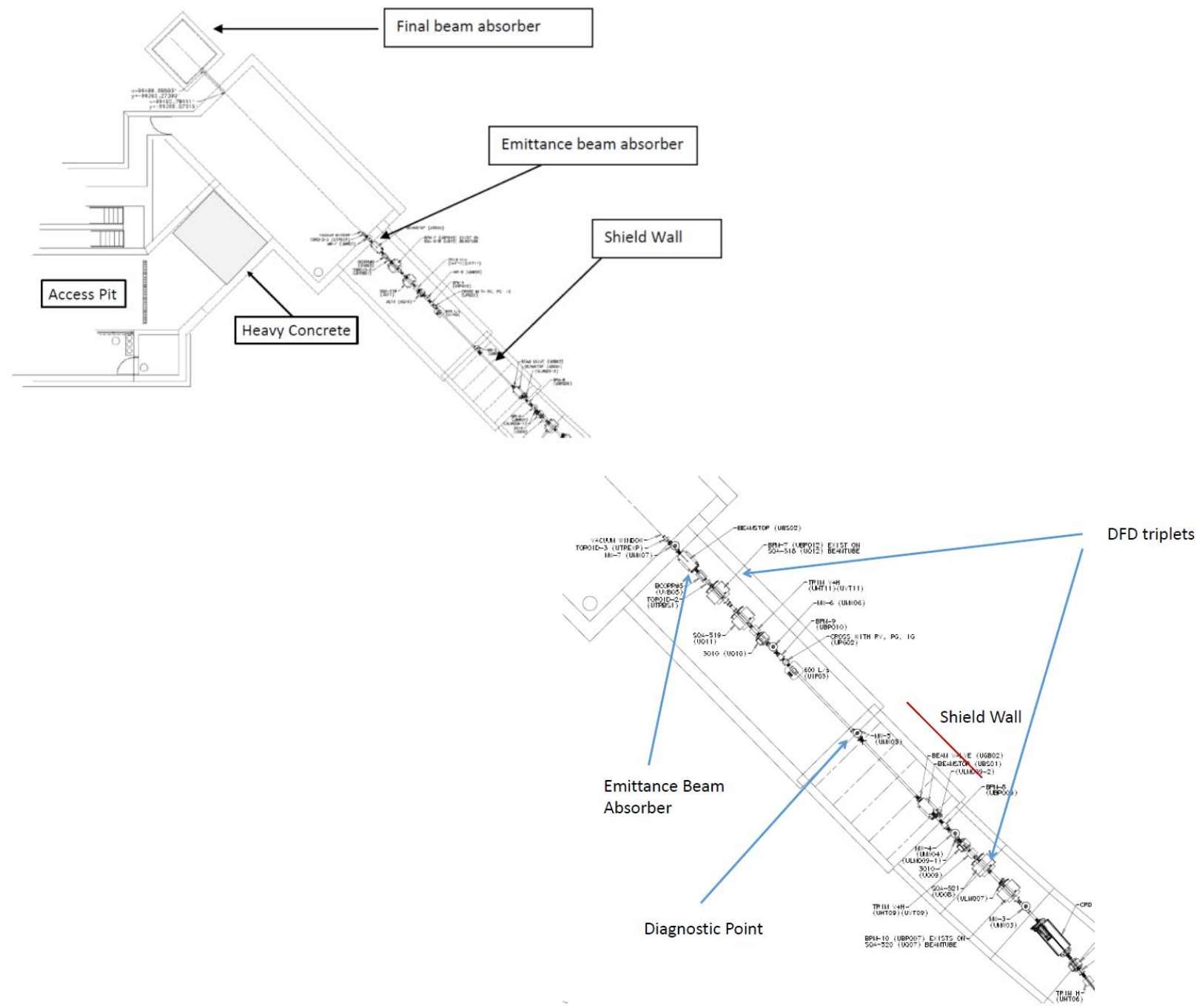

Figure 10. MTA layout downstream of the shielding wall (top), and MTA layout straddling the shield wall (bottom). Beam direction is from bottom right to upper left $[3,4]$.

1 Throughout this section discussion of existing intensity limits and shielding are obtained from refs. 16,22,23. 


\section{Emittance Mode:}

1) $9.6 \times 10^{15}$ protons/hr or 600 beam pulses/hour of full Linac beam pulse intensity $(1.6 \mathrm{x}$ $10^{13}$ protons/pulse) to the emittance beam absorber (see location of emittance absorber in Figure 1). The yearly integrated number of protons would be $2.3 \times 10^{17}$

In the Emittance mode beam is always deposited in the emittance beam absorber and the absorber is designed for $15 \mathrm{~Hz}$ operation at full Linac intensity (A thermal analysis was done). The allowed 600 pulses can be delivered sequentially in an hour which, in a thermal analysis, is essentially steady state so a higher number of pulses can be tolerated. Optics of the low-beta Linac diagnostic insertion are shown in Figure 11.

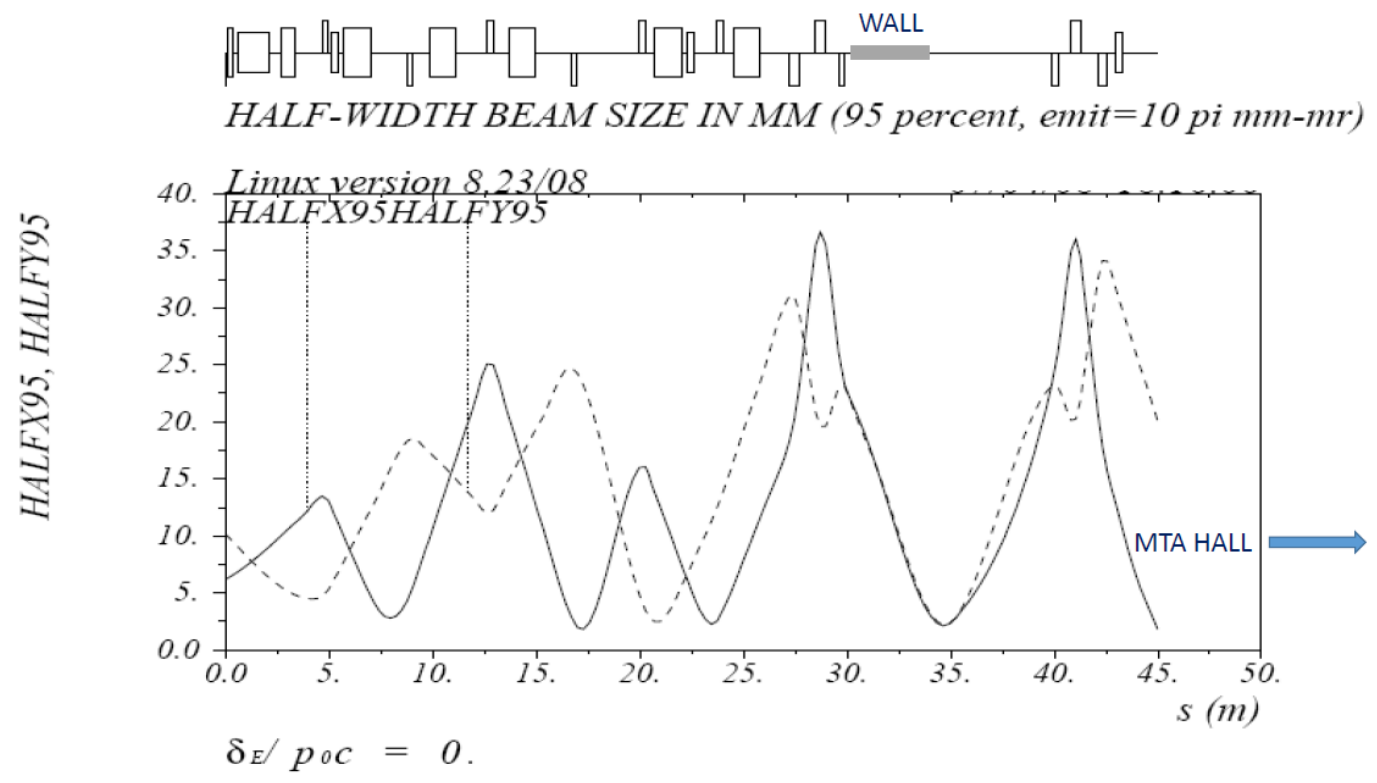

Figure 11. Low beta Linac diagnostic insertion in Emittance mode [3].

\section{Experiment Mode:}

2) $9.6 \times 10^{14}$ protons/hour or 60 beam pulses/hour of full Linac beam pulse intensity to experiments in the MTA experimental hall. In this mode, only $2.3 \times 10^{17}$ protons can be delivered yearly.

In the Experiment mode, two configurations are supported as illustrated in Figures 12,13.

a) Beam is cleanly transported in vacuum to the entrance of the high-intensity beam absorber shown in Figure 12. Not shown in Figure 12 are downstream magnetic components which 
will be needed to control beam delivery without losses to the absorber. To date beam has not been delivered to the high-intensity absorber.

b) The proton beam fully interacts in the experimental apparatus and the final beam absorber is not used. No downstream magnetic components are required for this configuration and this operational mode has been utilized for experiments.

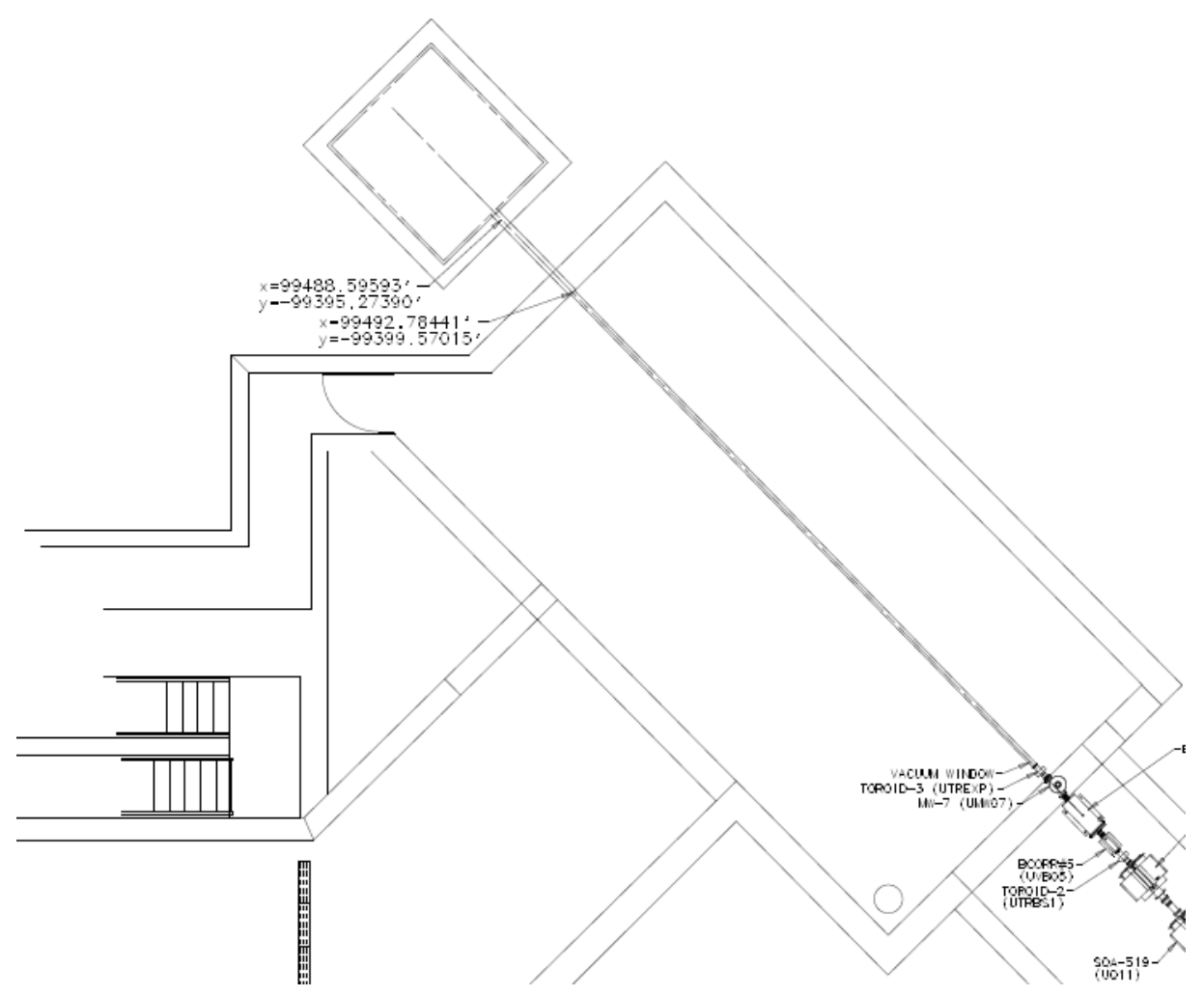

Figure 12. The experimental hall showing Experiment mode configuration a) with a vacuum pipe and no target in the hall. Beam can be cleanly transported by adjusting the focal length of the downstream quadrupole triplet [3]. 


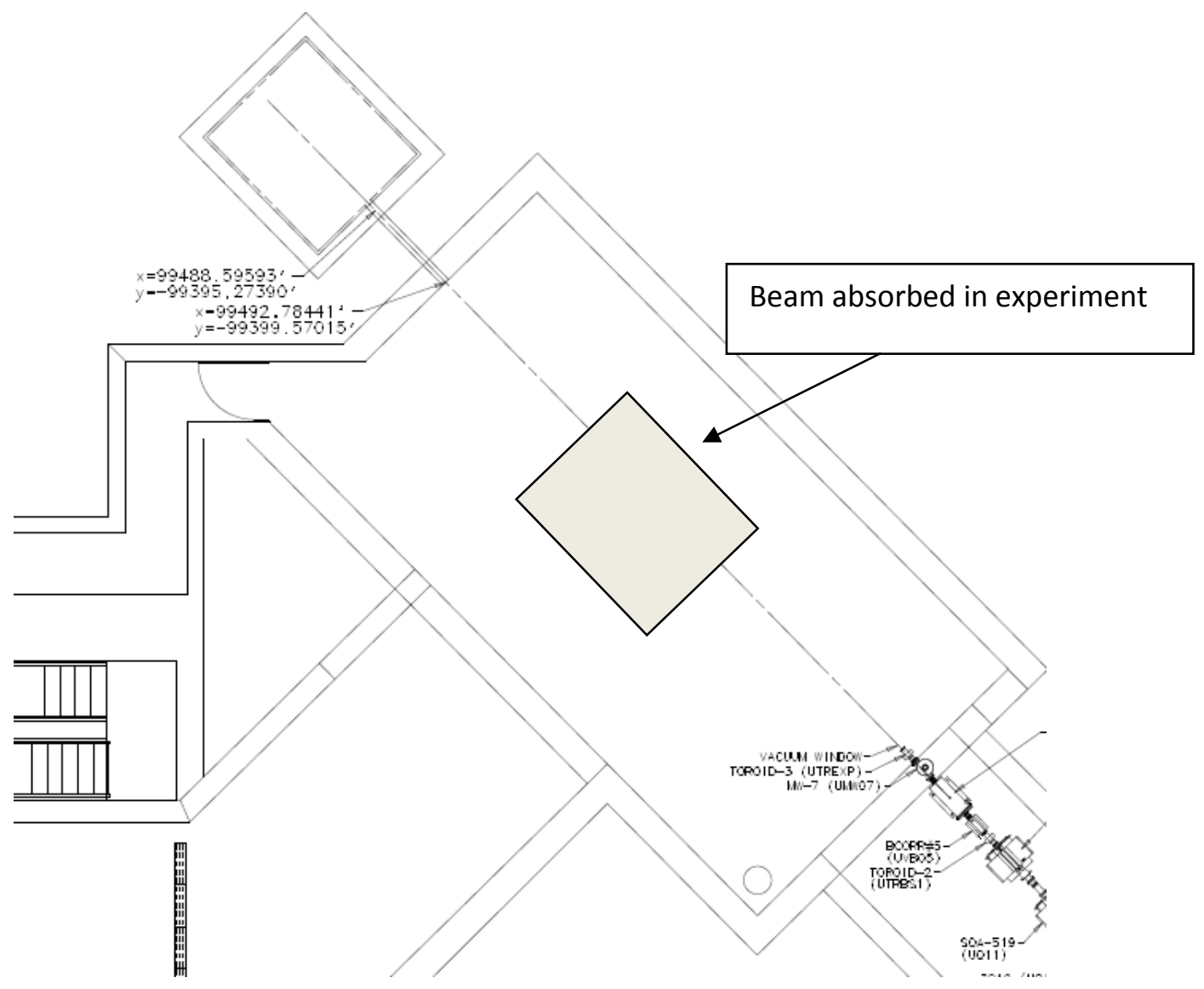

Figure 13. The experimental hall showing Experiment mode configuration with a $100 \%$ interaction length target in the hall so that beam is completely absorbed [3].

\section{High Intensity Mode:}

In its current configuration the MTA area cannot support high intensity beams. In the Emittance and Experiment modes the current shielding limitations restrict beam intensities to $\sim 0.4$ and $\sim 0.04 \mu \mathrm{A}$, respectively, which is too low to be competitive as a $\mu$ SR facility. In addition to adding MTA shielding - which could be local - it is clear that quadrupoles and steering trims in the hall would be required to transport Linac beam cleanly to the high intensity absorber as losses in the berm surrounding the beampipe to the absorber are not permitted. A simple layout of a quadrupole triplet formed with 200-MeV TQT quadrupoles is shown in Figure 14 for perspective. 


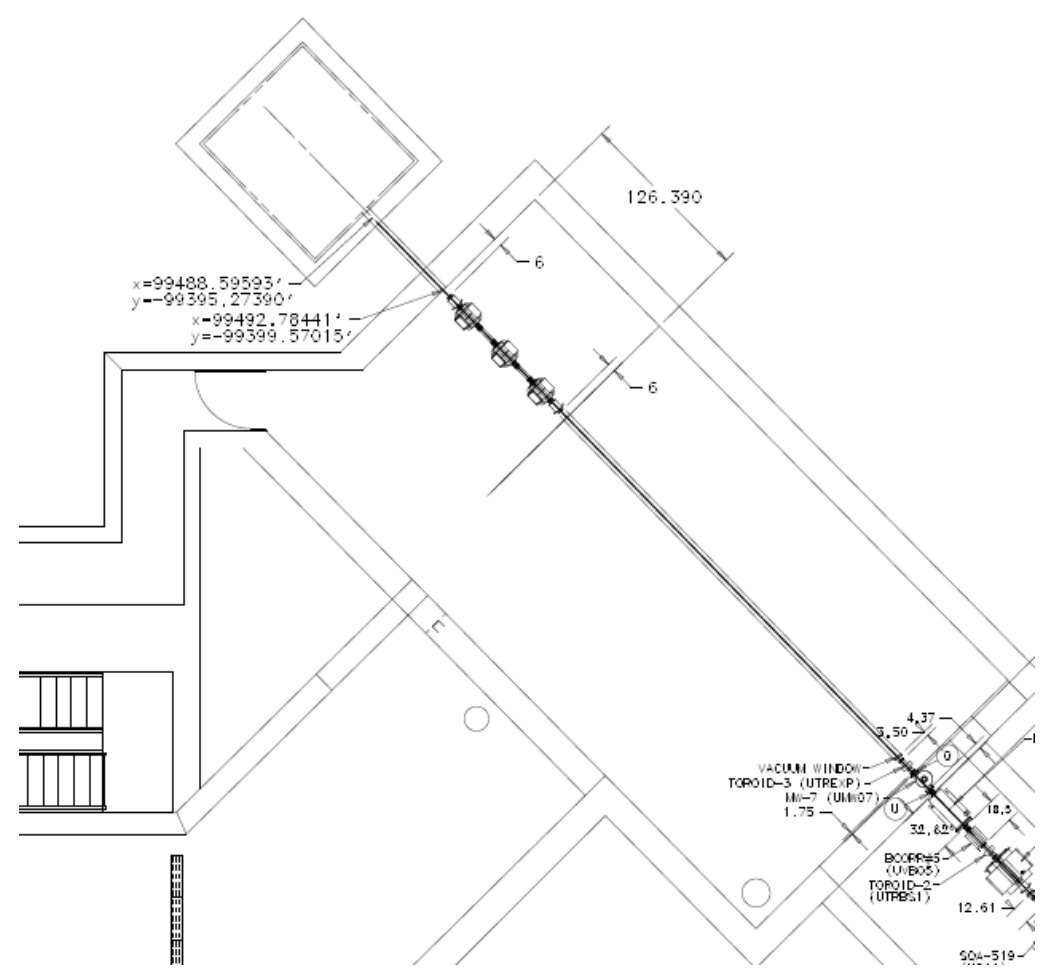

Figure 14. Conceptual example using a second downstream quad triplet of an experiment configuration which minimally interacts with beam (estimated to be $<10 \%$ of an interaction length) where beam has to be focused onto the final beam absorber [3].

\section{High Intensity $\mu$ SR Absorber}

Specifications for a new facility must include occupation of the experimental hall, hands-on maintenance requirements, residual activation of components, and a defining criterion by which a limit can be established on the total number of protons per year that can be delivered to the facility; i.e. running conditions. Normally the total number of protons delivered is dictated by the physics of an experiment, and highly-irradiated components, such as beamline elements and targets with hands-on restrictions are not normally a defining consideration but rather a shielding issue. Generally, there is low residual activation of experimental detectors, and, accesses, work, and occupation of the hall is often limited once the experiment is in data mode. This is not the case in a test facility where the total number of protons the facility can accept (operational envelope) must be specified using other criteria. Further, the occupancy of the hall may be frequent in such a facility with rotating experiments and maintenance on or change-out of irradiated components such as the muon production target may be potentially routine. The guidelines and administrative control over radiation and dose levels are more similar to those established for accelerator operation and maintenance, but potentially with the complication of higher occupancy levels. Below are rough guidelines that may apply to or must be considered with respect to a $\mu$ SR facility. 


\section{1) Hands-on Maintenance}

Administrative controls at FNAL limit the equivalent dose received by users and employees to $100 \mathrm{mr} /$ week. A surface-contact dose of $40 \mathrm{mr} / \mathrm{hr}$ requires increased supervision and monitoring by radiation control personnel. Limiting the surface-contact dose to $40 \mathrm{mr} / \mathrm{hr}$ (in the experimental hall) and carefully simulating the $\mu$ SR target and beamline (using MARS) the activation of components can be calculated as a function of the total number of protons for full Linac intensity. The point at which an exposed surface reaches $40 \mathrm{mr} / \mathrm{hr}$ can be used to set the upper limit to the total number of protons which the facility can receive in a year. The operational limit may be mitigated by low-activation shielding installed to protect beamline components and instrumentation. Commonly used at Fermilab are 4" marble slabs which can reduce residual dose by factors up to $\sim 100 x$.

\section{2) Occupancy Limits}

Work areas (which should include the majority of the hall) must be $<5 \mathrm{mr} / \mathrm{hr}$, which is the lower limit for a radiation area. Discussed in a later section, this limit has significant impact on the design of the absorber at the beam exit of the experimental hall.

\section{3) Total Number of Protons per Year}

The limit on the total number of protons per year can be set by activation of components at levels tolerable for hands-on maintenance. There is a sample simulation that was performed for MuCool targets which might suggest an estimate for the total allowable protons on target (POT) per year and is discussed in the following section.

\section{4) Beam Parameters}

The beam parameters are, and must be, defined almost completely by clean transmission to the beam absorber after the $\mu$ SR target. That is, what are the optics that essentially allow $99 \%$ of the beam to be captured and cleanly transported to the high-intensity beam absorber. An important question is whether adequate optics can be implemented within the $\sim 40$ ' of the experimental hall, and, if not, does one compromise the emittance measurement section which is contained in the "stub" section. A preliminary optics solution is studied for feasibility in a later section.

\section{MARS Simulation and Results for MuCool Apparatus}

A full MARS simulation was performed on the MuCool hydrogen absorber for a 400-MeV (kinetic energy) incident proton beam of $10^{13}$ protons per pulse at $15 \mathrm{~Hz}$ (four $0.2 \mathrm{~mm} \mathrm{Al}$ windows and $21 \mathrm{~cm}$ of liquid hydrogen surrounded by a superconducting NbTi solenoid) [24]. Important to this feasibility study, power density, absorbed dose, and residual dose were calculated for downstream beamline components and particle fluxes in the experimental hall. Little sensitivity to a variation in beam size was found. These simulations are important here in that they guided the design of the high-intensity beam absorber and set initial limits on the number of protons/year and the most effective irradiation and cool-down scenarios. The POT limit is unlikely to 
significantly impact the prompt residual dose in the experimental hall, but will impact activation of components in the hall which were found to be more sensitive to beam irradiation and cooldown scenarios discussed next.

\section{Activation and Residual Dose}

Residual dose results were computed for 30 days of beam and 1 day of cooling for $10^{13}$ protons per pulse at $15 \mathrm{~Hz}$ for various elements of the MuCool apparatus and beamline components. From this simulation for the peak dose on contact not to exceed $40 \mathrm{mr} / \mathrm{hr}$, a total beamtime of 100 days of full Linac beam, or $10^{21} \mathrm{p} /$ year on MuCool apparatus, was found to be the POT limit. It should be noted that fewer days of continuous running did not result in a corresponding reduction factor in activation and was approaching a "steady-state" activation level. Running for a day with an extended cooling period of a few days was found to be optimal for minimizing residual dose rates in components (Figures 15, 16). This past study may provide approximate guidelines in suggesting an operational template for a $\mu$ SR facility. One number from the absorber simulation remains valid - the absorber core activation of $9000 \mathrm{mr} / \mathrm{hr}$. This simulation was also cautionary in that it yielded high activation of downstream beamline components, clearly indicating the need for protective collimators to shadow these components.

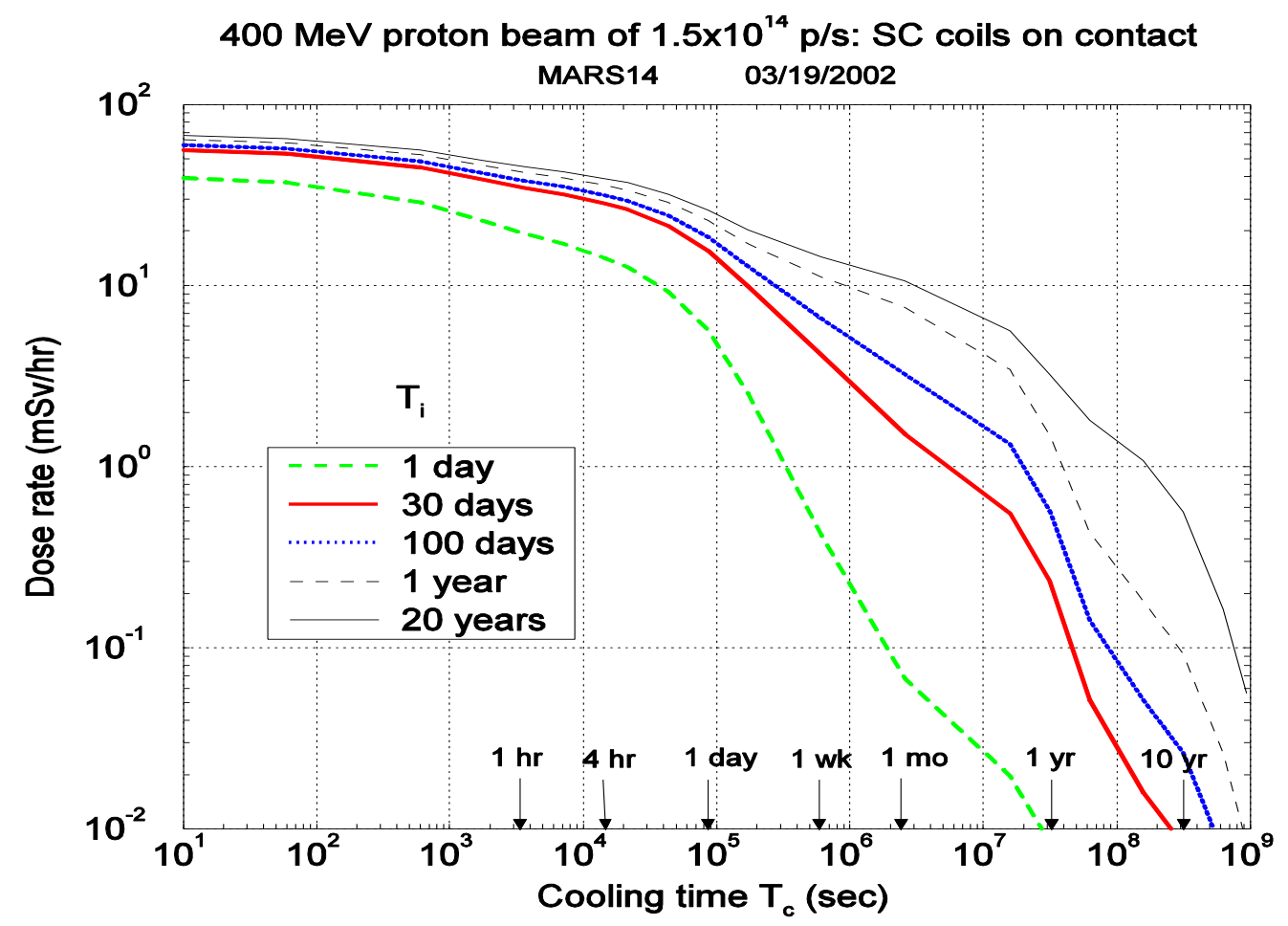

Figure 15. Residual dose and cooling rates for SC coils on MuCool solenoid. This was used to estimate surface dose vs irradiation scenarios. 
This simulation is also cautionary from the perspective that it yielded high activations of downstream beamline components - several hundred $\mathrm{mr} / \mathrm{hr}$ levels for 30 days of running on MuCool apparatus and 1 day of cooling - indicating the possible need for protective collimators to shadow these components. Particular attention needs to be paid to shielding the $\mu$ SR target. Although the target is anticipated to be a 5\% instead of $100 \%$ nuclear interaction length, the activation even if the results are simply scaled are high - approaching $50 \mathrm{mr} / \mathrm{hr}$. Marble is a lowactivation material and sufficient space is provided between components to insert protective marble to shield cores and coils. The caveat is that simulations were performed for the worst case, a $100 \%$ nuclear interaction length target.

The MuCool experimental hall has a load capacity of 19' equivalent of dirt overburden, but only $\sim 10.5^{\prime}$ of berm was implemented for a total height of $12.5^{\prime}$ [22]. The reduced height from $19^{\prime}$ to $12.5^{\prime}$ 'was required to match to the 11 ' of berm consistent with upstream Linac enclosure shielding and elevation level. The initial shielding report [23] indicated heavy concrete was intended to be installed to reduce radiation levels at the service of the berm as shown in Figure 17 (dose rate from 0.25 up to $5 \mathrm{mrem} / \mathrm{hr}$ [25]). This was not done and a radiation fence was installed instead around the MTA berm. Alternatively, local concrete shielding can be installed and 6' gains approximately two orders of magnitude in intensity. There is 6' available radially about a target location (the beamline center is more than 6' off the enclosure floor), but a careful assessment is required to verify a local shielding design.

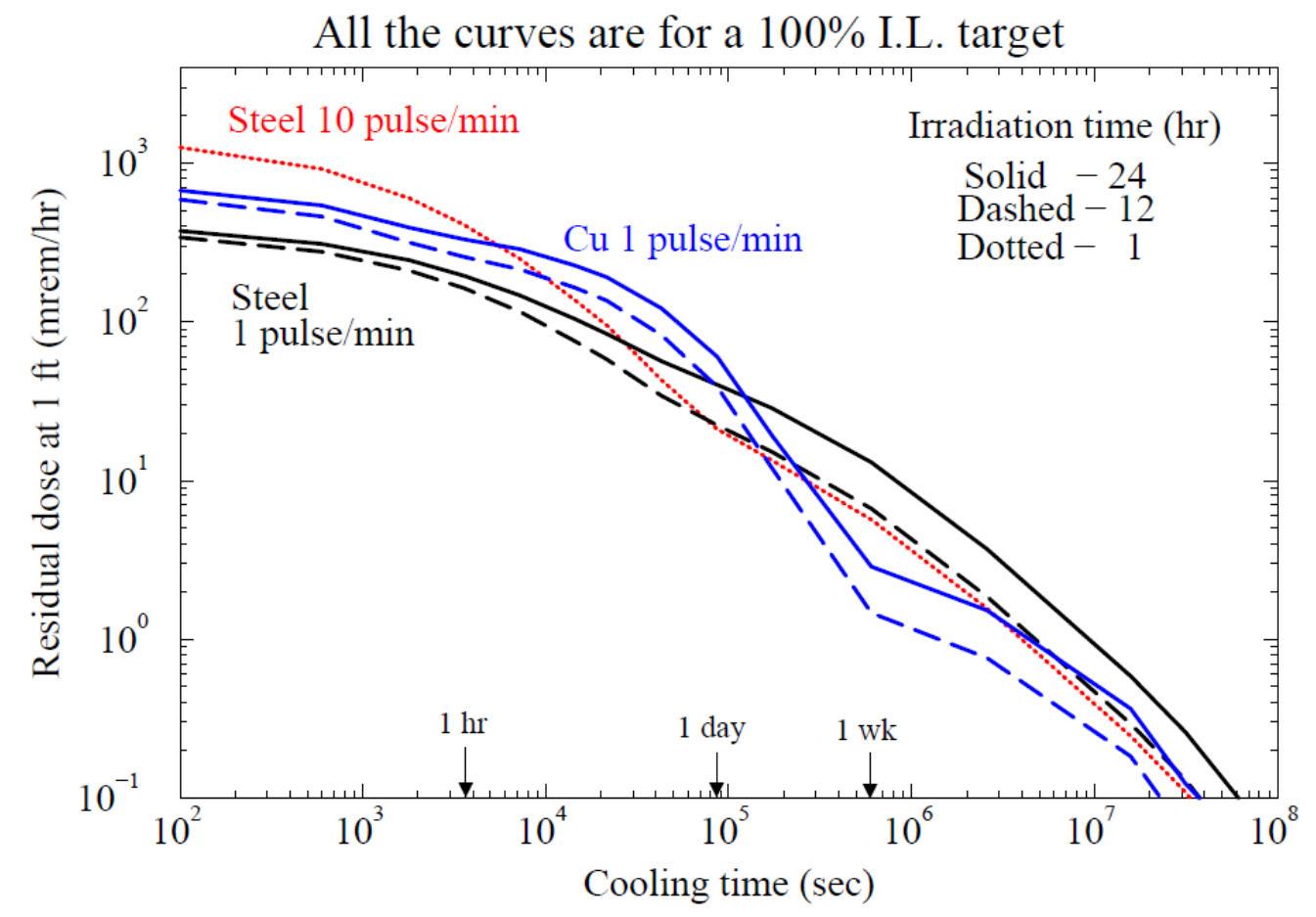

Figure 16. The calculated potential residual dose at one foot for Emittance and Experiment mode, and $1.6 \times 10^{13}$ p/pulse, on $100 \%$ interaction length $\mathrm{Cu}$ and steel targets 
for 12, 24, and 1 hour periods of beam followed by a cooling period. A $100 \%$ interaction length target represents the worst case [22]
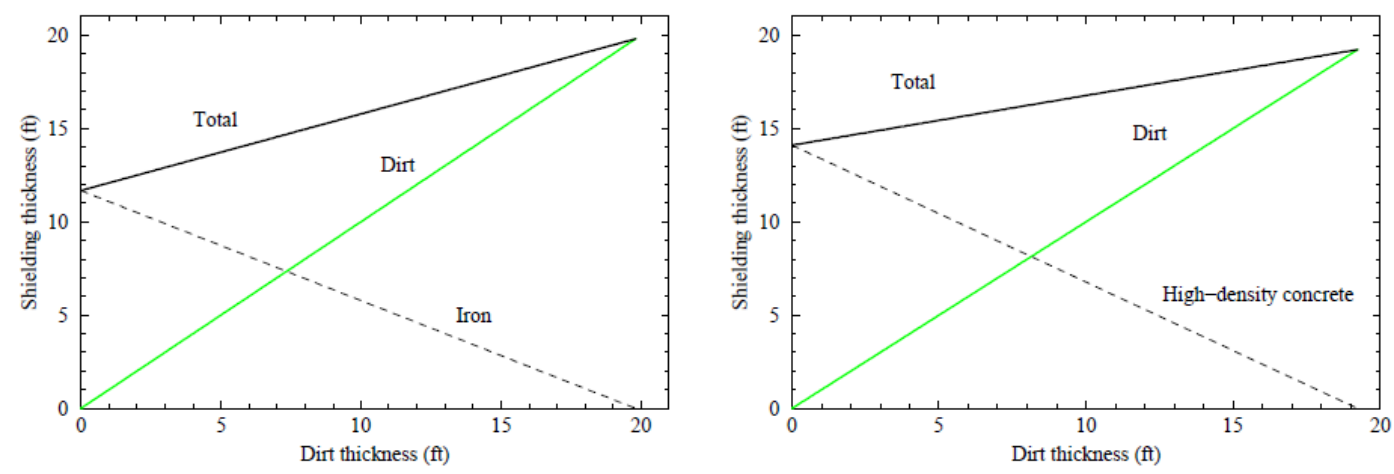

Figure 17: Calculated shielding compositions for iron-dirt (left) and BMCN-dirt (right) sandwiches which provide the dose level of $0.5 \mathrm{mrem} / \mathrm{hr}$ on the top of the MTA shielding at normal operation [23].

\section{Beam Absorber Design and Simulation}

The absorber was designed to support this upper limit of $10^{21}$ protons per year in terms of ground and surface water contaminants and surrounding shielding, but the design also limits the prompt residual dose to the experimental hall to the $<5 \mathrm{mr} / \mathrm{hr}$ limit. Attenuation of the prompt residual dose was accomplished by restricting the opening aperture of the absorber to 4" and offsetting the absorber by about 6' from the experimental hall. Prompt residual dose in experimental hall after 100 days of irradiation and $0.5 \mathrm{hr}$ of cooling time was $8 \mathrm{mr} / \mathrm{hr}$ at $4^{\prime}$ offset from the entrance of the absorber and decays by about a factor of 3 for every 2' in this region. (The entrance of the absorber is about $240 \mathrm{mr} / \mathrm{hr}$.) Although hitting the soil shielding around a buried berm pipe is prohibited, a larger (10") pipe increased the prompt residual dose at 4" to 20 $\mathrm{mr} / \mathrm{hr}$ (approximately in the ratio of the pipe radii). Figure 18 below shows the MARS model of the absorber for prompt residual dose in the hall and for calculation of star densities, which are shown in the subsequent figure. 

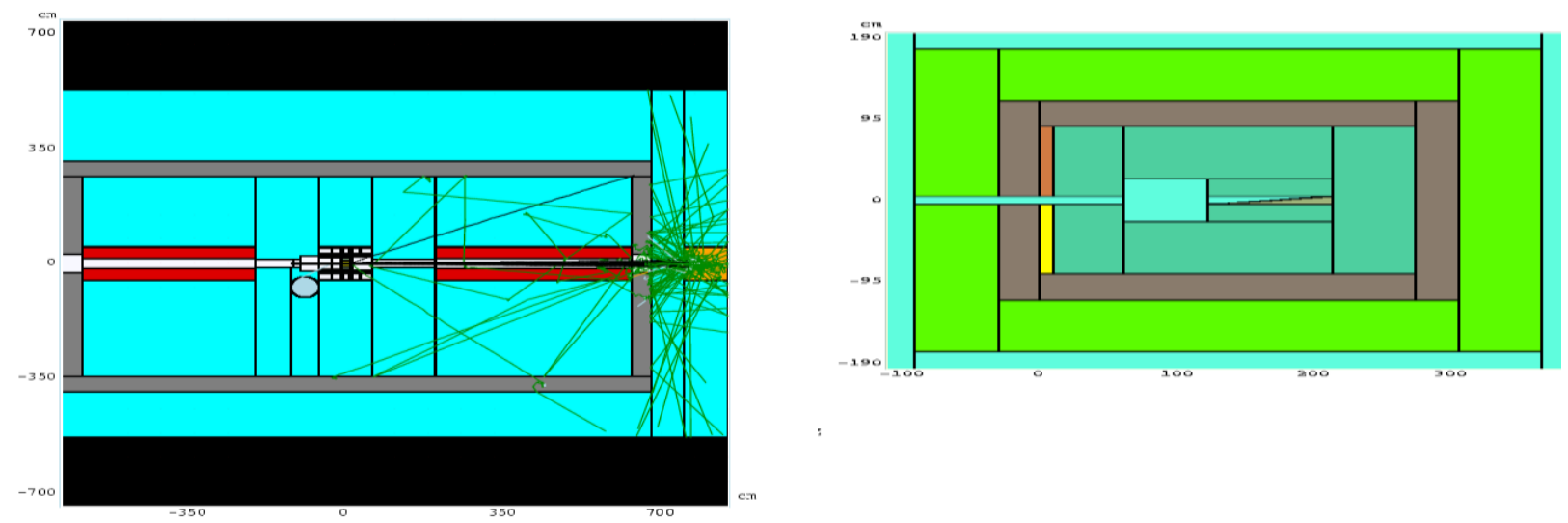

Figure 18. Particle trajectories in the experimental hall, beam on (left). Layout and dimensions of the MTA beam absorber (right). An absorber aperture/berm pipe of 4" diameter and offset by more than 5 ' brought the prompt residual dose to levels less than a defined radiation area in the experimental hall. The inner $\mathrm{Cu}$ wedge (right) is a solid billet, surrounded by steel, concrete and soil, respectively. The light turquoise represents the albedo trap and beam pipe.

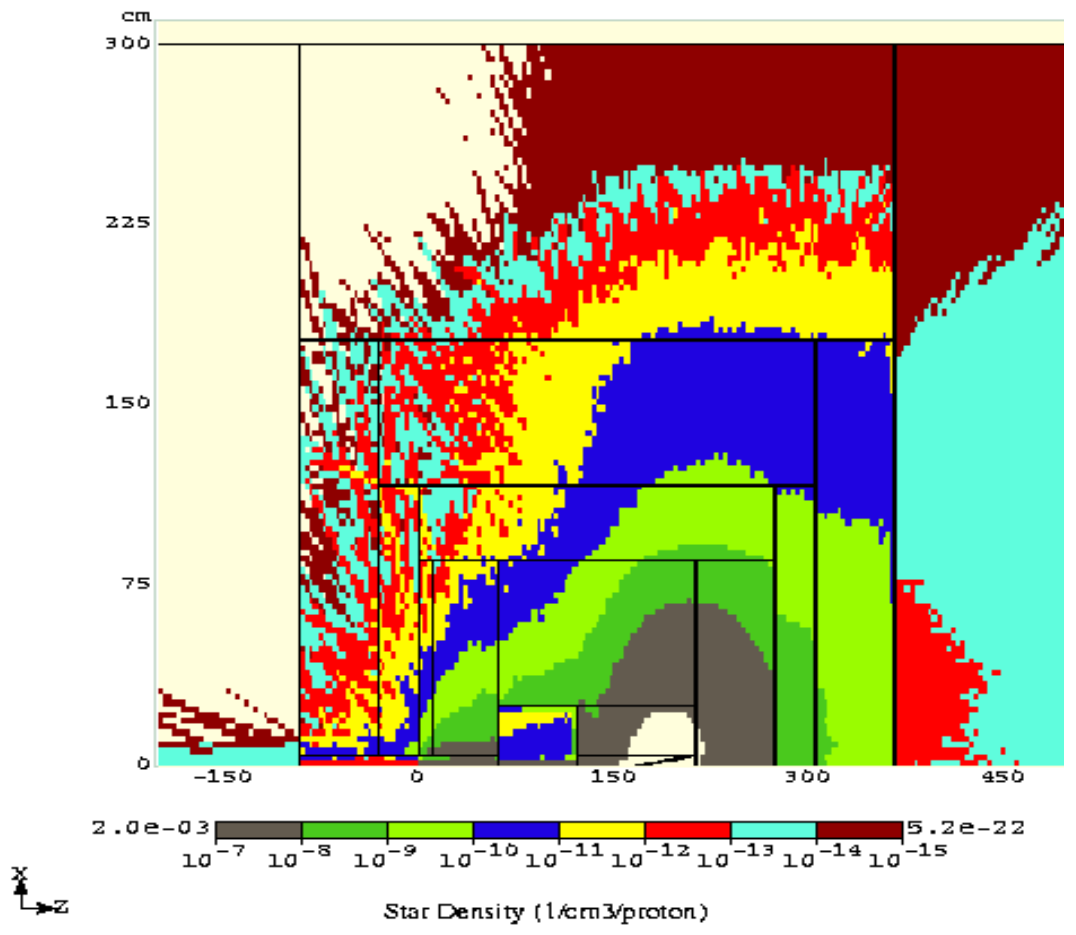

Figure 19. MARS calculated star density distribution. The coordinate system begins at the front face of the steel at the entrance to the absorber. In the region between about 0 and $50 \mathrm{~cm}$ and 225 and $275 \mathrm{~cm}$ horizontally and 0 and $75 \mathrm{~cm}$ vertically lies the steel surrounding the core. The core itself lies between about 125 and $225 \mathrm{~cm}$ horizontally and 0 and $25 \mathrm{~cm}$ vertically. The steel is followed by about $18 \mathrm{~cm}$ (1') of concrete and then soil or fill begins less than -25 and 
greater than $300 \mathrm{~cm}$ horizontally and above $115 \mathrm{~cm}$ vertically. A schematic view of the beam absorber is provided in Figure 20.

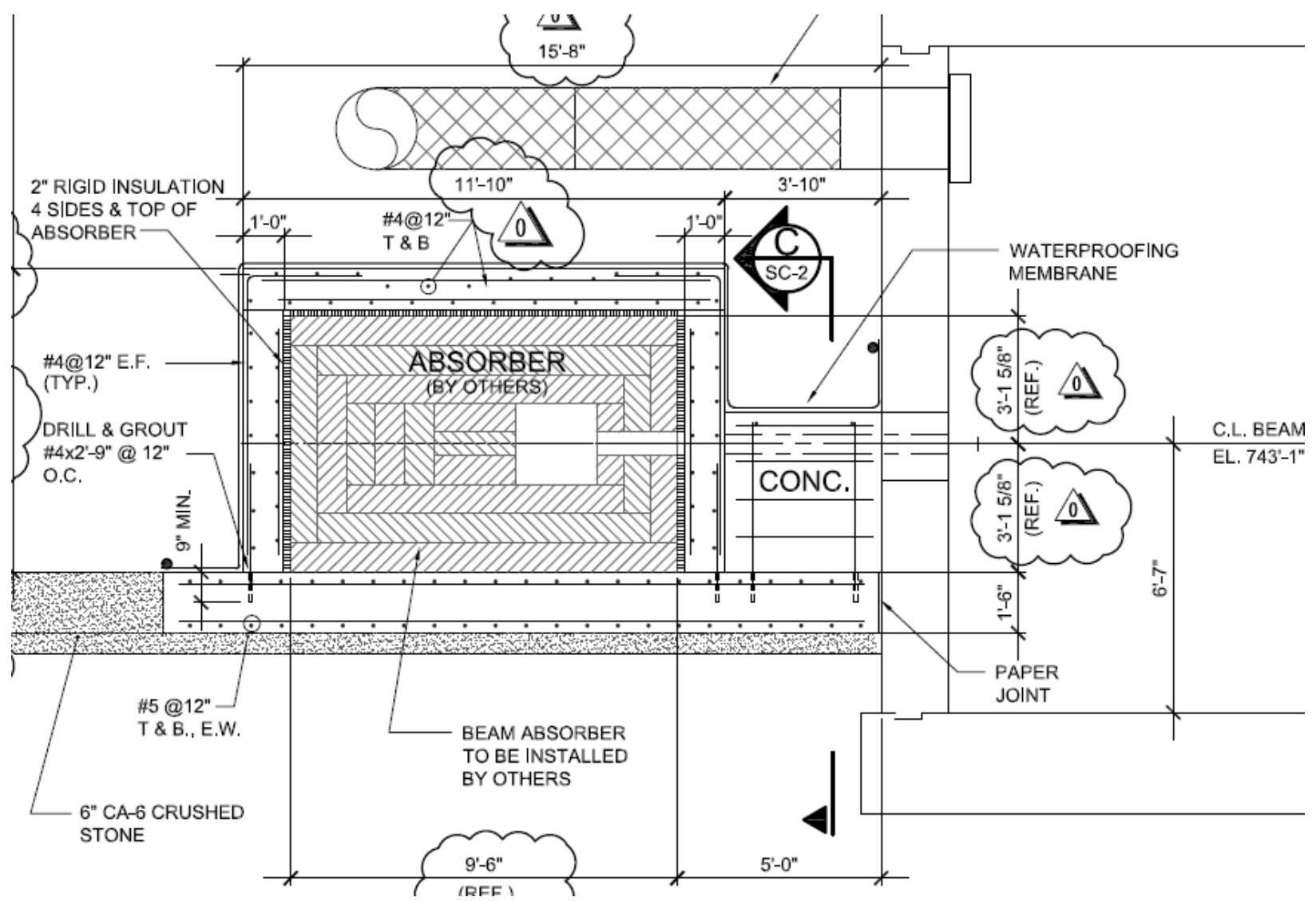

Figure 20. Civil plan layout of the beam absorber showing $\sim 6.5$ ' offset from exit of experimental hall.

\section{Beam Absorber Design}

The main features of the beam absorber were designed to meet combined operational and occupancy criteria and include:

1) Sufficient steel to attenuate the star density in the soil surrounding the beam absorber by 8 orders of magnitude as can be seen in the star density calculation of Figure 19.

2) 22' equivalent of soil has been maintained from the beam spot in the absorber to the surface of the berm to drop radiation levels to unlimited occupancy rates;

3) A copper core was necessary to dissipate and distribute the power of the full Linac beam $(10 \mathrm{~kW})$. This was confirmed by several ANSYS analyses of different core configurations. 
4) An "albedo" trap or void was designed into the beam absorber to reduce high neutron fluxes back propagated into the experimental hall during beam (a factor of 10 reduction).

5) An absorber aperture/berm pipe of 4" diameter and offset by more than 5' brought the prompt residual dose to levels less than a defined radiation area in the experimental hall.

The basic layout of the final beam absorber is shown in Figure 20. A 6" copper billet, 24" long makes up the core of the beam absorber and is press fit into the surrounding steel. This core is surrounded by varying lengths of 8 " by 8 " flame-cut steel. The entire absorber is encased in 1' thick concrete. A minimum of 5 ' of soil separates the front of the beam absorber from the experimental hall. The detailed ground and surface water calculation are included in a separate section in the report of ref [16]. To insure slow diffusion of water around the absorber and ultimately into the aquifer, there is no opening to the drain tiles which surround the experimental hall in the vicinity of the beam absorber thereby isolating the sump system from drainage around the beam absorber. The beamline vacuum terminates in a vacuum window at the entrance to the beam absorber. There is no need to extend the vacuum into the interior of the absorber. (Thermal properties of the absorber core have been simulated for very small beam spots, $<1 \mathrm{~cm}$, and maximum intensity to verify thermal stability under the worst possible beam conditions.)

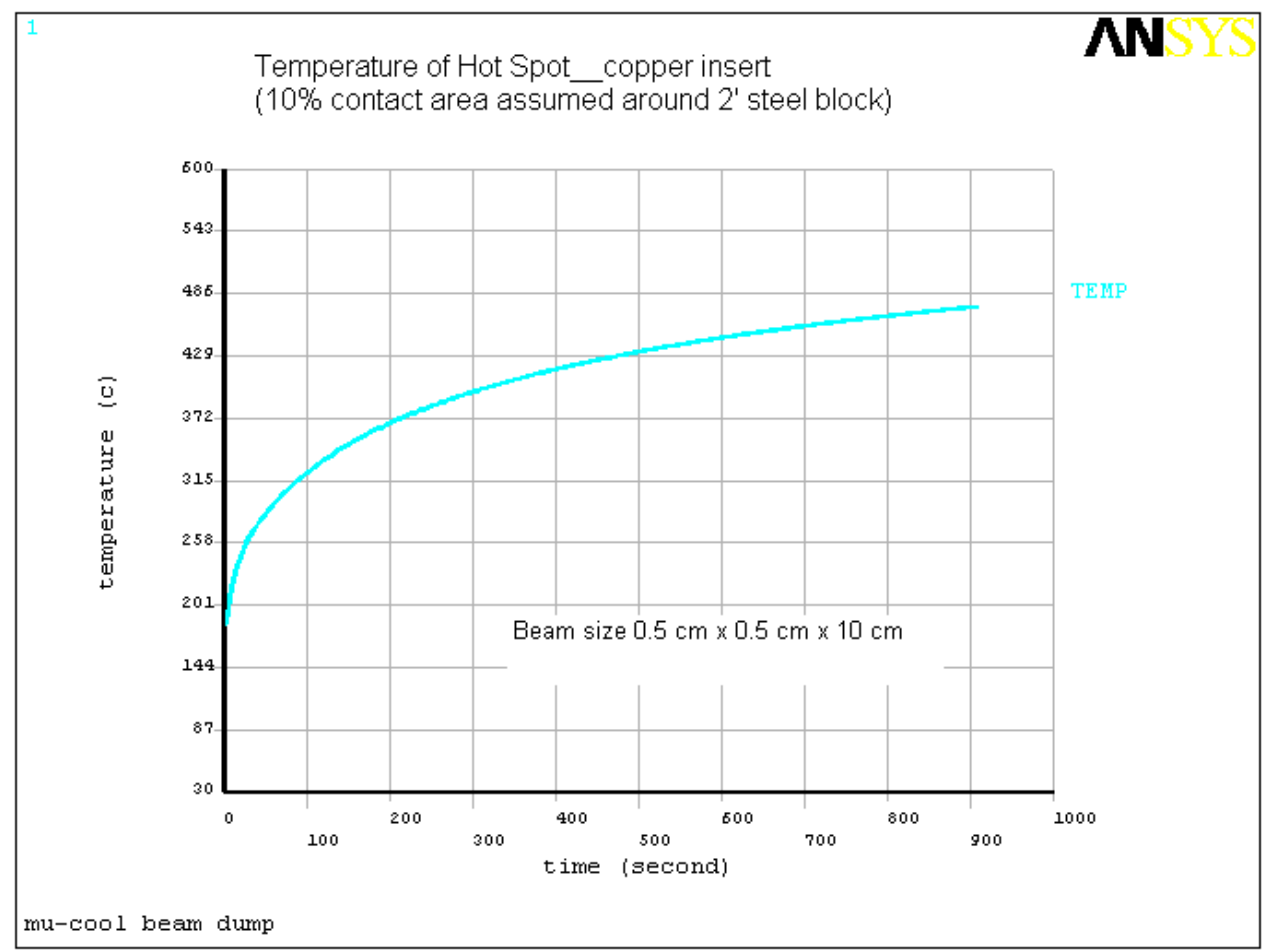

Figure 21. Temperature history of the center hot spot of the absorber [17] - melting point of copper is $1085^{\circ} \mathrm{C}$. 


\section{Ground and Surface Water Activation Calculations}

The limit for tritium, ${ }^{3} \mathrm{H}$, and for sodium- $22,{ }^{22} \mathrm{Na}$, in surface water is $2000 \mathrm{pCi} / \mathrm{ml}$ and 10 $\mathrm{pCi} / \mathrm{ml}$, respectively. For ground water the corresponding limits are $20 \mathrm{pCi} / \mathrm{ml} 0.4 \mathrm{pCi} / \mathrm{ml}$, respectively. The assumptions and the calculations of the star density for the high-intensity beam absorber are given in reference [26]. No granular underdrainage was installed in this region to keep activated water out of the Booster sump system and force water to dissipate into the soil around and below the absorber. Surface water limits, therefore, are not relevant to the absorber. Ground water limits POT to approximately $2.01 \times 10^{20}$ per year for the sodium.

Activated air is also released; $30 \mathrm{Ci}$ in a year from the MuCool Facility was determined by the ES\&H to be part of the integrated Fermilab yearly site discharge allowed. However, based on air activation released from the existing MuCool cryogenic operations, the maximum number of protons/year was reduced to $2.35 \times 10^{18}$ and further required an exhaust requirement of $1200 \mathrm{cfm}$. If cryogens are not needed in future experiments and the exhaust fan flow rate reduced to less than $80 \mathrm{cfm}$, the number of protons/year can be increased to the sodium limit.

\section{Beam Production Optics for $\mu S R$}

A preliminary optics design for $\mu \mathrm{SR}$ has been performed in order to assess if a beamline was feasible in terms of beam capture and transport to the high intensity absorber and if the required length can be accommodated within the limited $40^{\prime}$ available in the experimental hall. The target could in principle be placed in the stub upstream of the hall adding additional length, but transverse space is limited. Additional local shielding about a high-intensity target would present access and equipment aisle conflicts in addition to the absence of crane coverage. Further, the stub would not allow a muon beamline to be established at $90^{\circ}$ or upstream relative to the target as is the norm to avoid swamping muon detection with background. Exploiting or reconfiguring the shield wall that separates the MTA area from the Linac enclosure is not feasible from a shielding, operational, or cost consideration. (The shield wall is located under and was stacked through an overhead hatch.) At this time a target location in the stub is not a feasible option.

Given the high-intensity requirements for a $\mu \mathrm{SR}$ experiment, the beamline optical and component design must achieve better than $99 \%$ transmission of the secondary beam to the highintensity absorber. The tails of the beam - outside of the 2.5-3 $\sigma$ definition - can be collimated upstream but beam at the downstream wall of the experimental hall must approach a 3"-diameter size and maintain this size, or less, throughout the vacuum section. Once inside the beam absorber, the beam profile can clearly be allowed to expand. The 4"-diameter beam pipe extends 6.5 ' from the exit of experimental hall (5' of berm pipe and 18" of concrete enclosure wall) to the opening in the concrete that defines the entrance of the dump. Control over and stability in the beam optics 
of the absorber is critical to avoid irradiating the soil that surrounds the pipe, especially important at high intensity. Since losses up to the entrance to the albedo trap should be considered to avoid backscattering and neutron reflection into the soil, the total beam confinement length increases to $101.88^{\prime \prime}\left(8.5^{\prime}\right)$ or $2.6 \mathrm{~m}$. In order to constrain the beam size to be less than or equal to the beam spot at the downstream wall, a beam focus must be maintained near the center of the beampipe section in the soil.

A minimum beam envelope, not just a centrally-located focus, should be established over the entire 102" length to maximally protect the surrounding soil from contamination. It can be easily shown that the minimum envelope across a drift entails a $\beta=1 / 2 \mathrm{~L}$ waist at the center of the drift and $\mathrm{L}$ is the length of the drift. Rounding up to $3 \mathrm{~m}$, this implies a low $\beta$ waist of $\sim 1.5 \mathrm{~m}$ located halfway between the exit of the hall and the albedo trap entrance. Upstream and downstream, the beta function reaches a maximum of $2.6 \mathrm{~m}$ again at the exit of the hall and at the entrance to the Albedo trap.

Then, from restricting apertures, a total emittance that can be transported cleanly can be calculated. For 4" pipe (beam tails should be collimated upstream) a \pm 1.5 " beam should be cleanly transmitted. However, the nominal aperture of $400-\mathrm{MeV}$ quadrupoles is 3.25 " which is more restrictive and quadrupoles always represent the highest beta locations. To characterize required optics, beta functions must be assumed at the $\mu$ SR target. For this purpose, a simplification of a low-beta waist at the center of the $\mu \mathrm{SR}$ target is used to start a design which can be propagated to the absorber and then iterated. The emittance blows up at the target, which can be calculated but, more importantly, the impact of the target can be represented by a discrete jump in the beta function given the emittance blowup and upstream waist beta functions. Given that the emittance blowup from the target scales proportionally to the beta function, the lowest beta waist that achieves the physics objectives should be implemented. To avoid aberrations common with ultra-low betas, a low $\beta=1 \mathrm{~m}$ is used as a starting point to design preliminary optics downstream of the target. (Beta functions after a target reduce by approximately the ratio of the pre- to post-target emittances and a very low beta is problematic for optics stability; similar to a collider IR). For the purposes of an initial beamline design, $2 \mathrm{~m}$ is used between the target and first quadrupole, an inter-magnet drift of $0.25 \mathrm{~m}$, and $0.5 \mathrm{~m}$ is inserted between the last quadrupole and the downstream enclosure wall. The last drift will be important for shielding to collimate the remaining tails of the beam and protect the berm from production products and plume from the $\mu \mathrm{SR}$. Four quadrupole magnets in the experimental hall downstream of the $\mu \mathrm{SR}$ target are required to achieve the correct beam absorber optics regardless of production beam properties as there are four beam properties to match $-\beta_{x, y}$ $=1.5 \mathrm{~m}$ and $\alpha_{x, y}=0-$ at the focal midpoint inside the berm. Dispersion is canceled in the linac part of the beamline and both $\mathrm{D}$, and $\mathrm{D}^{\prime} \sim 0$ are good assumptions and consistent with the small spot sizes already achieved and measured in the MTA hall.

A preliminary optics design is shown in Figure 22 which is capable of clean transmission to the beam absorber for the largest emittance blowup achievable as restricted by the present berm pipe aperture. Downstream magnetic components will require shielding from secondary particles but sufficient insertion space has been incorporated. The maximum beta in the 4 " berm pipe is 
about $3 \mathrm{~m}$ (5m to $6.5 \mathrm{~m}$ is halfway from the exit of the experimental hall to the entrance of the albedo trap).

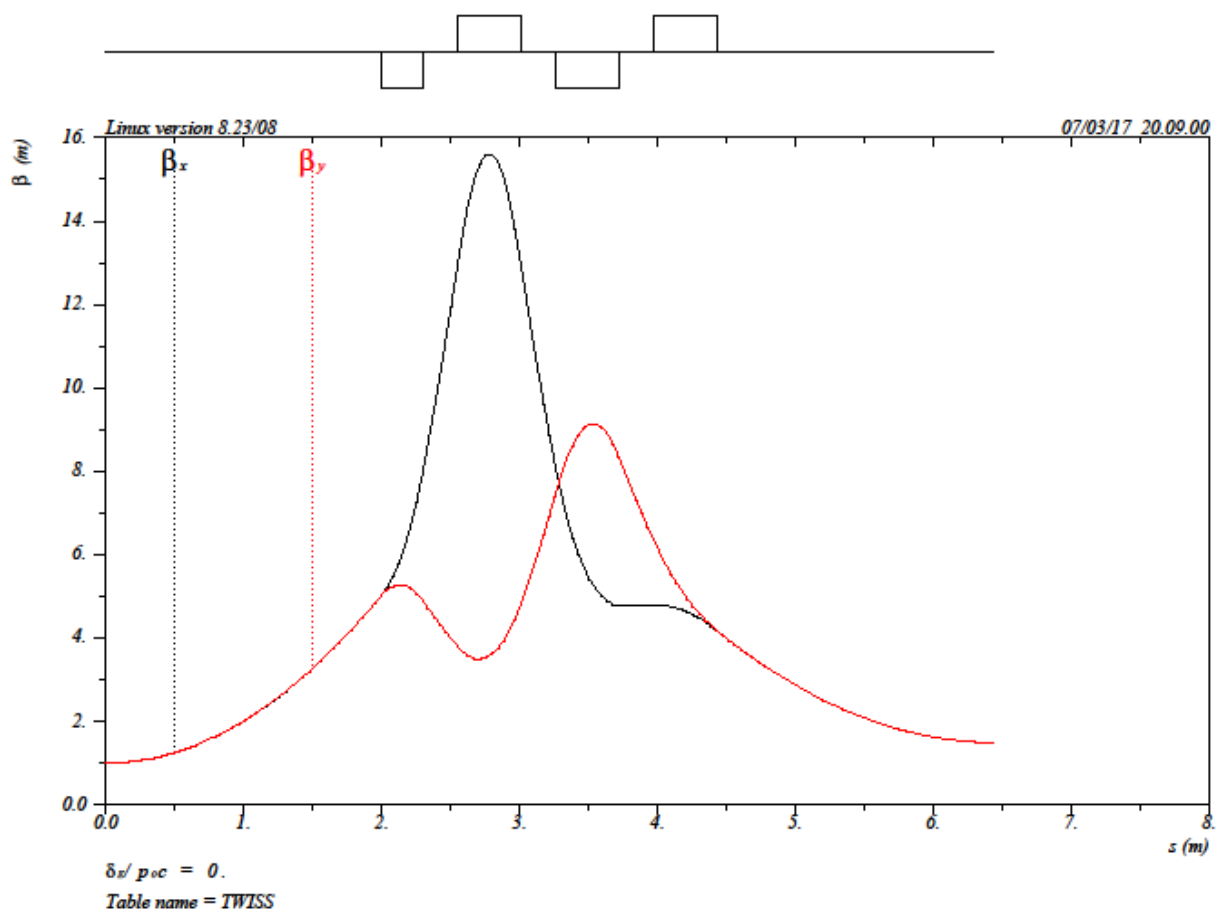

\section{Figure 22. Optics from $\mu$ SR target to midpoint of drift to absorber albedo trap}

These starting optics can be used to determine the maximum emittance that can be cleanly transported to the absorber. The Linac beam is well represented by a Gaussian and beam tails outside of $2.5 \sigma$ can be scraped by collimators to prevent and insure minimal losses inside the berm pipe. The acceptance, or maximum emittance, assuming 0.25 " beam-stay-clear, is then determined using \pm 1.75 " to calculate a $2.5 \sigma(95 \%)$ beam size for clean transmission. For comparison, the maximum beta function of $\sim 15 \mathrm{~m}$ in the transport optics and a large aperture (8") quad at that location is also calculated.

$$
\begin{gathered}
2.5 \sigma=\sqrt{\beta \varepsilon} ; \beta=3 \mathrm{~m} \text { and } 2.5 \sigma=44.45 \mathrm{~mm}\left(\frac{1}{2} 3.5 "\right) \\
\sigma=17.78 \mathrm{~mm} ; \epsilon=659 \pi \mathrm{mm}-\mathrm{mr} ; \\
2.5 \sigma=\sqrt{\beta \varepsilon} ; \beta=15 \mathrm{~m} \text { and } 2.5 \sigma=95.25 \mathrm{~mm}\left(\frac{1}{2} 7.5^{\prime \prime}\right) \\
\sigma=38.1 \mathrm{~mm} ; \epsilon=635 \pi \mathrm{mm}-\mathrm{mr} ;
\end{gathered}
$$


(normalized $\varepsilon \sim$ unnormalized $\varepsilon$ for $400 \mathrm{MeV}$ protons)

This estimate is tricky because the optics downstream of the target must be re-initiated with a change in beta function. For a waist at a thin target (this is an approximation but surprisingly good), the post-target low beta must scale as the ratio of pre-target to post-target emittances in order to maintain the same spot size in order to continue use the Courant Snyder functions. A small spot size, an approximate point source, focused on the target not only minimizes the emittance blowup from multiple scattering, it also produces a new phase-space ellipse that can be propagated and is target independent (independent of final post-target emittance). However, the new low beta of the phase-space ellipse is limited to 1 meter to maintain stable envelope control.

The Linac beam is approximately $8 \pi \mathrm{mm}-\mathrm{mr}$ for $3 \sigma$ [21]. For the present design, this allows a large emittance blowup, in theory more than a factor of 50 based on the estimates above. (Again note that a localized change in emittance results in a corresponding decrease in the beta function to maintain the same spot size which will in turn change the peak beta functions in the transport line). In order to design the transport line, a downstream starting low beta of $1 \mathrm{~m}$ must first be fixed and the upstream section of the line tuned to produce the corresponding pre-target beta function. A low beta below $1 \mathrm{~m}$ is difficult to control and produces higher and higher peak transport betas reducing the acceptance of the channel - or quadrupoles must be moved close to the target and close to one another eliminating the ability to install inter component shielding.

Establishing the final post-target low-beta is sufficient to predesign the transport optics to the absorber, it is not sufficient to model the target and pre-target conditions. Although one can estimate the emittance blowup and corresponding multiple scattering angles that can be tolerated based on aperture restrictions, it turns out that does not result in an acceptable target for muon production. One needs to start from a target which is estimated to be about $5 \%$ of a nuclear interaction length $\left(\lambda_{\text {nuc }}\right)$ to make a competitive facility - ISIS, for example is a $4 \%$ target. The following derivation starts with a $5 \% \lambda_{\text {nuc }}$ target and works backward through the target based on the emittance blowup ratio of target beta functions to derive pre-target optical conditions. For the following Be will be used because the ratio of radiation length to nuclear interaction length is 1.2 which makes for smaller multiple scattering and downstream emittance blowup versus graphite or nickel targets. Emittance blowup is the limiting factor for muon production at the MTA facility.

$$
\begin{gathered}
\theta_{r m s}=\frac{13.6 \mathrm{MeV}}{p v} \sqrt{\frac{l_{\text {tgt }}}{L_{\text {rad }}}}\left[1+0.038 \ln \left(\frac{l_{\text {tgt }}}{L_{\text {rad }}}\right)\right] \text { for Be } l_{\text {tgt }}=0.05 \lambda_{\text {ruc }}=1.2 L_{\text {rad }} \\
\therefore \theta_{\text {rms }}=4.38 \mathrm{mr} \text { for } B e
\end{gathered}
$$


The emittance blowup in terms of the upstream, $\beta_{\mathrm{u}}$, and downstream, $\beta_{\mathrm{d}}$, beta functions is given by the following equation. Again, the lowest downstream "stable" beta function is fixed to be $1 \mathrm{~m}$ in order to not only calculate the optics but also to minimize the emittance blowup.

$$
\begin{gathered}
\Delta \epsilon=3 \beta_{u} \theta_{r m s}^{2}=3 \beta_{d}\left(\frac{\epsilon+\Delta \epsilon}{\varepsilon}\right) \theta_{r m s}^{2} ; \text { for } \beta_{d}=1 m \text { post target } \\
\Delta \epsilon=3\left(\frac{\epsilon+\Delta \epsilon}{\varepsilon}\right) \theta_{r m s}^{2} \\
\text { so }: \epsilon=\frac{3 \theta_{r m s}^{2} \Delta \epsilon}{\Delta \epsilon-3 \theta_{r m s}^{2}} ;
\end{gathered}
$$

note that emittance blowup $\Delta \epsilon>3 \theta_{r m s}^{2}$ or there is no solution;

for a general parameterization: $\epsilon=\frac{\chi \Delta \epsilon}{\Delta \epsilon-\chi}$ with $\chi=3 \theta_{r m s}^{2}$

for $\Delta \epsilon=595 \pi$ from above and $\theta_{r m s}=4.38 \mathrm{mr}$ for a $5 \% \lambda_{\text {nuc }}$ target

$$
\chi=57.55 \text { and } \Delta \epsilon=10.3 \chi
$$

this equation becomes $\epsilon_{u}=\frac{1}{9.3} \Delta \epsilon=64 \pi m m-m r ; \epsilon_{d}=659 \pi m m-m r$;

so the incoming emittance, $\epsilon_{u}$, must be $\sim$ a factor of 8 larger; $\beta_{u}=\frac{659}{64}=10.3$

The conclusion is that a 5\% target will not work for a Be target unless the Linac beam emittance is first increased by a factor of 8 - as derived later, an $8 \pi \mathrm{mm}-\mathrm{mr}$ beam limit is a $1.5 \%$ $\lambda_{\text {nuc }}$ target, which is not sufficient. A graphite target would require a lower $\lambda_{\text {nuc }}$ given its larger ratio of nuclear $\lambda_{\text {nuc }}$ to radiation length $\lambda_{\text {rad }}$, but lithium has a larger ratio than beryllium and might be a very interesting muon production candidate. (A combined lithium lens and target might also provide muon collection and capture, but this is purely speculation at this point.)

Increasing the emittance by a factor of 8 can be achieved upstream given the low energy of the beam and by assuming a simple vacuum window - a 5 mil Ti window, for example, which has a high radiative length and a low nuclear interaction length, and back calculate the beam optics required. Using the multiple scattering equation to find the rms scattering angle for a $4 \mathrm{mil} \mathrm{Ti}$ $\left(\lambda_{\text {rad }}=3.56 \mathrm{~cm}\right)$ gives

$$
\theta_{r m s}=0.94 \mathrm{mr}
$$

A factor of 8 blowup in emittance from 8 to $64 \pi \mathrm{mm}-\mathrm{mr}$ gives the following equation:

$$
\Delta \epsilon=3 \beta \theta_{r m s}^{2}=56 \pi m m-m r ; \beta_{u}=21.1 m ; \beta_{d}=2.6
$$


Incoming beam on the window is then tailored to a waist of $21.1 \mathrm{~m}$ which becomes $2.6 \mathrm{~m}$ post window. These optics can be ideally generated in the $5 \mathrm{~m}$ component free section downstream of the location of Multiwire \#5 (MW5), which is located just downstream of the shield wall (Figure $23)$. The $2.6 \mathrm{~m}$ beta waist is then propagated to the muon target which, for an incoming $64 \mathrm{~mm}$ $\mathrm{mr}$ emittance, the scaling factor is approximately $(659) / 64=10.3$, thereby allowing the $\beta$ to change from a 1meter waist at the muon production target for the downstream absorber optics to a $10.3 \mathrm{~m}$ waist upstream of the target; this waist which must be generated by intermediary and secondary upstream optics. (The part of the beamline between the Linac and this waist is normally designated as a primary beamline.) Fortunately, such a waist can be achieved using the existing downstream triplet, UQ10-UQ12, originally designed to provide a varying beam focus for a broad range of locations and experimental requirements in the hall, plus two additional quadrupoles located in the experimental hall just downstream of the stub (the stub has a $2.5^{\prime}$ ' concrete step level change to the hall for reference).

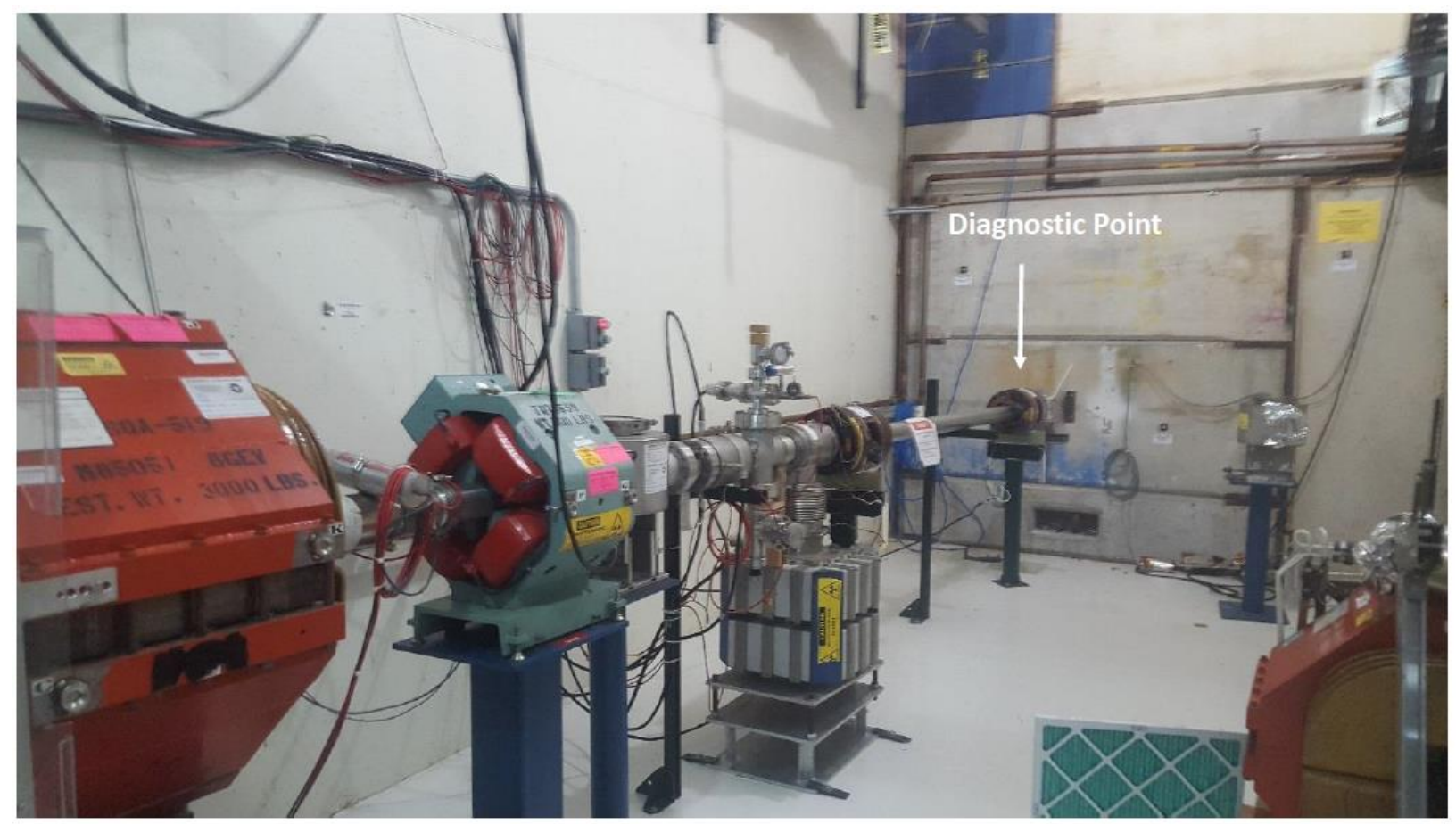

Figure 23. Looking upstream towards the shield wall and the location of MW5 near where a Ti window would be installed.

In Figures 24 and 25 below are plots of the optics for the different sections. A dispersionfree dual vertical and horizontal waist of $10.3 \mathrm{~m}$ is generated at the assumed Ti window location is provided by the upstream section of the line, UQ01-UQ09. The downstream $2.6 \mathrm{~m}$ waist is then propagated to a $10.3 \mathrm{~m}$ waist at the muon target using UQ10-12, a varying drift, and two additional quadrupoles, UQ13 since more than 4 variables are needed for matching to the 4 Courant Snyder parameters. After the preliminary design, an additional quadrupole was added to move the $\mu$ SR target further downstream from the stub, constrain the beam envelope to $\beta=15 \mathrm{~m}$ or less, and to 
allow some variation of the beam spot size on the muon production target. The $1 \mathrm{~m}$ beta function from the $\mu$ SR target is propagated to the opening in the absorber albedo trap. Collimators will be needed downstream of the target, shielding for the downstream magnetic elements and at the end of the beamline to protect the berm surrounding the absorber beampipe from secondary particles.

Importantly, the quadrupole triplet upstream of the emittance absorber plus two additional quadrupoles downstream of the absorber are able to generate the waist beta functions required at the $\mu$ SR target. This is of enormous benefit to fitting the target and downstream components within the experimental hall - approximately $8 \mathrm{~m}$ presently from the downstream enclosure wall. The experimental target will be located roughly a few meters from the edge of the stub (exit of the emittance absorber). If the target is just downstream of the beginning of the rollup door and its outside shield wall, then a $90^{\circ}$ muon beamline could exit through this shielding potentially allowing more available and possible critical shielding from hall backgrounds (Figure 26). A backward muon line could also be designed although space would be limited to 20 ' given the upstream presence of the stub. As the shielding assessment now stands, a prototype target could be implemented immediately but at the lower duty cycle of a pulse/minute although 60 pulses in an hour can be taken sequentially at the $15 \mathrm{~Hz}$ Linac rate as the hourly limit is the radiological figure of merit. This would allow different target materials and geometry to be evaluated.

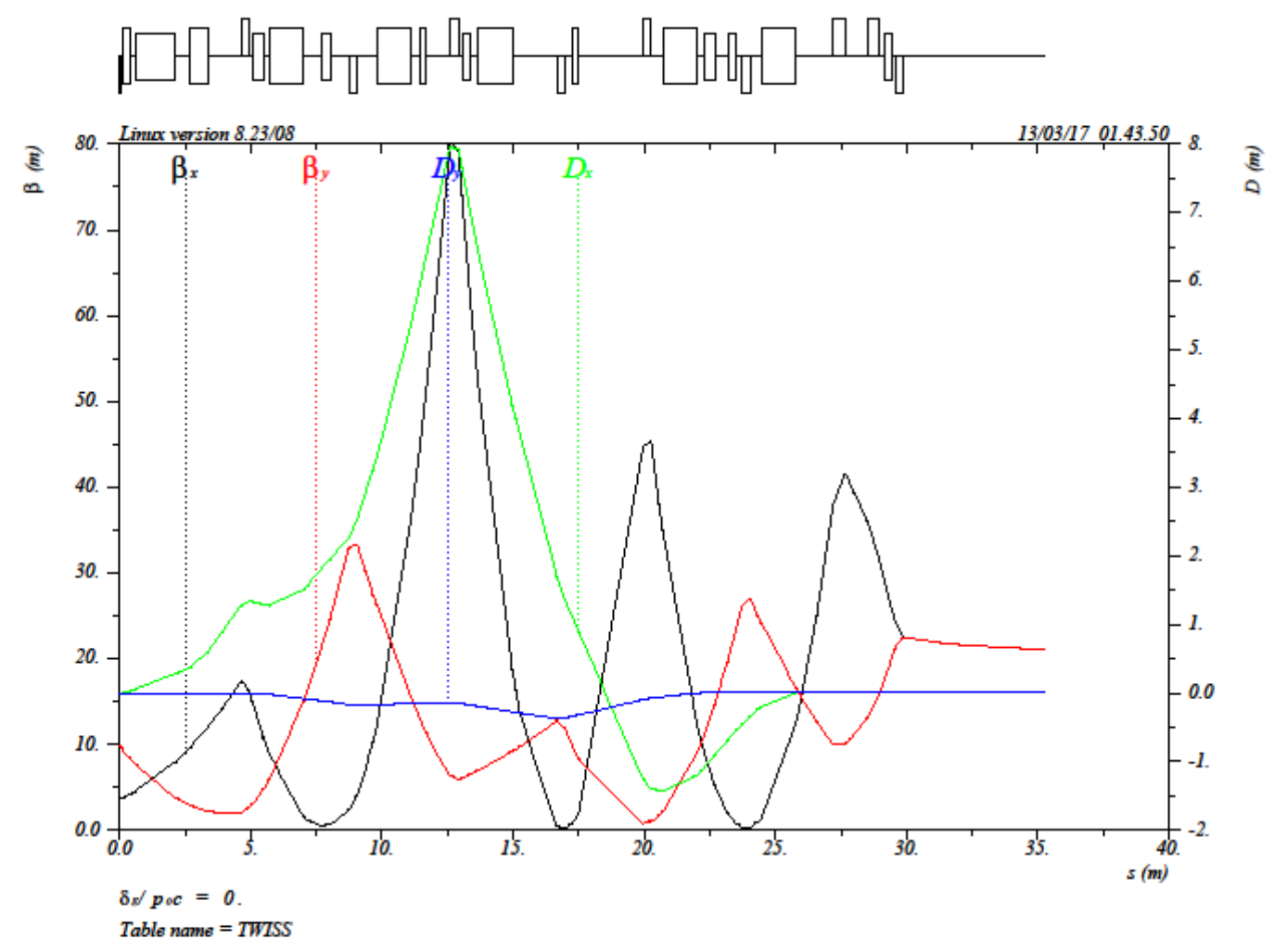

Figure 24. Optics from Linac extraction to emittance target downstream of shield wall. 




Figure 25. Preliminary optics from emittance target to $\mu$ SR target.

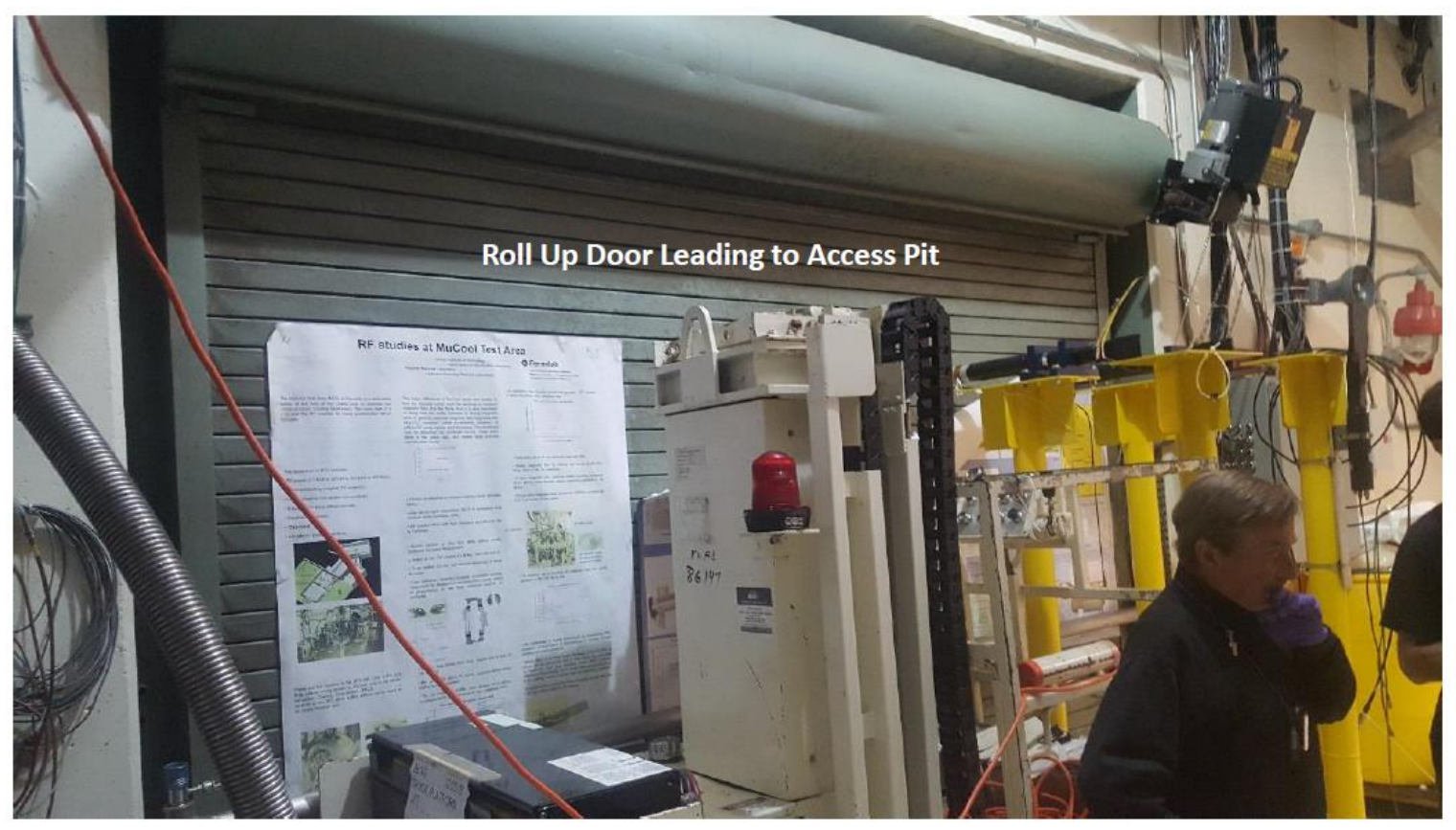

Figure 26. View of the rollup door in the experimental hall 
Although not a precise representation of the present layout (which requires 4 quadrupoles in the hall downstream of the target and two upstream), a previous optics design does show quadrupoles in the experimental hall which gives a sense of the dimensions and feasibility of such a beamline. The $\mu$ SR target preliminary location is marked for reference in Fig. 27. Components currently in the downstream end of the MTA beamline are shown in Fig. 28.

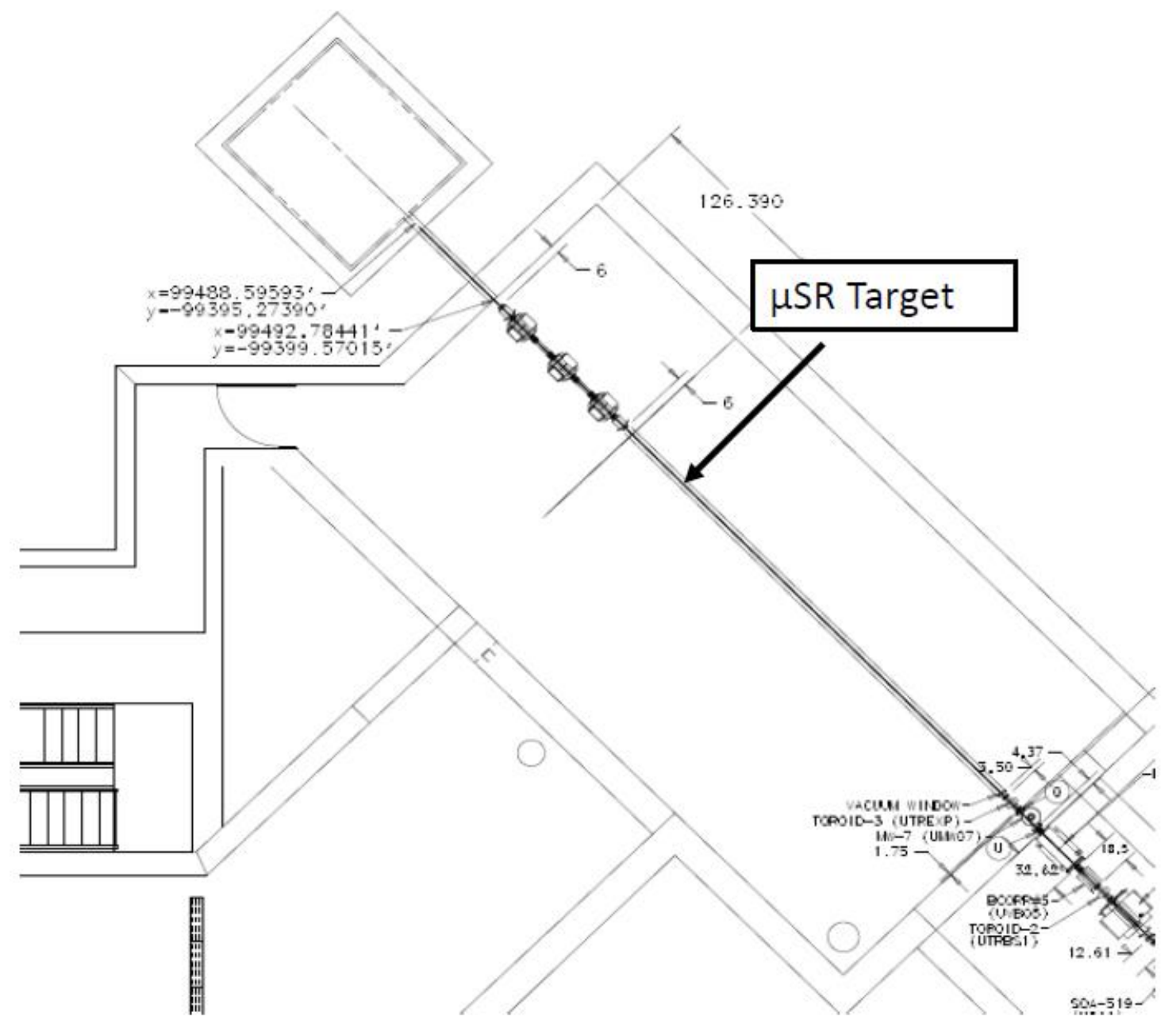

Figure 27. Layout of the experimental hall showing approximate potential location of the $\mu \mathrm{SR}$ target for this very preliminary design 

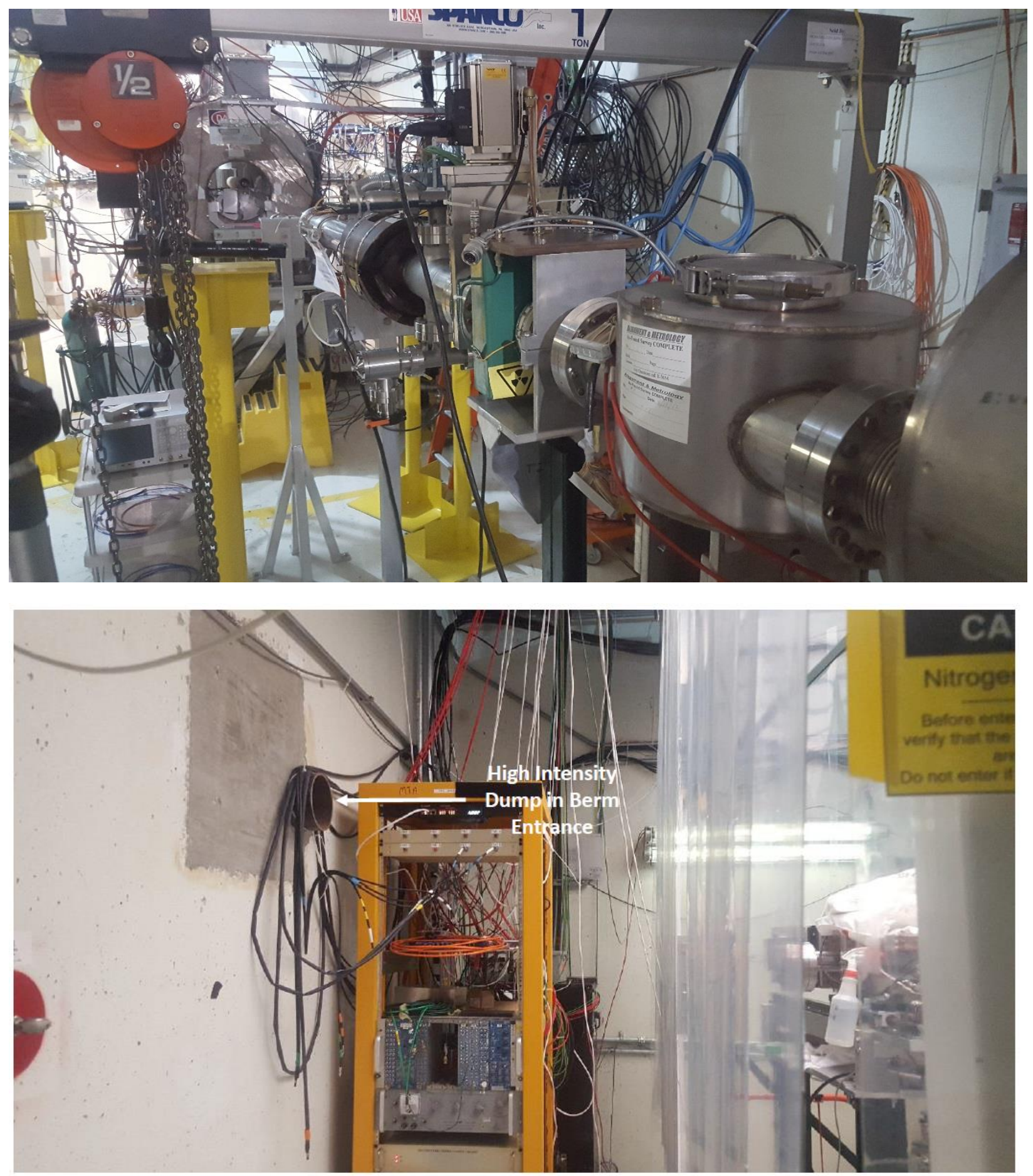

Figure 28. View downstream of the experimental hall (top) and high intensity dump entrance (bottom). 


\section{Single Target Production}

Alternatively, one can solve for the target limit for a single target production scenario. Starting with limiting the net change in emittance to $595 \pi \mathrm{mm}-\mathrm{mr}$ for the purposes of consistently calculating the allowable interaction length of the target based on the equations for multiple scattering. With a post-target beta of $1 \mathrm{~m}$, and an emittance ratio of $8 / 603$, then the incoming beta-function is exceptionally large, $75 \mathrm{~m}$. For a $95 \%$ beam the rms scattering angle can be calculated from the following:

$$
\begin{gathered}
\Delta \epsilon=3 \beta \theta_{r m s}^{2}=595 \pi \mathrm{mm}-m r ; \text { for } \beta=75 \mathrm{~m}, \theta_{r m s}=1.6 \mathrm{mr} \\
\text { where } \theta_{r m s}=\frac{13.6 \mathrm{MeV}}{p v} \sqrt{\frac{l_{\text {tgt }}}{L_{r a d}}}\left[1+0.038 \ln \left(\frac{l_{\text {tgt }}}{L_{\text {rad }}}\right)\right]
\end{gathered}
$$

A target length which is only $1.5 \%$ of radiation interaction length yields $1.5 \mathrm{mr}$ for $\theta_{\text {rms }}$. and less for a nuclear interaction length - for Be it is only $1.25 \%$ of a nuclear interaction length. This value for a nuclear $\lambda_{\text {nuc }}$ is not competitive with other facilities.

\section{Beamline Component Inventory}

A major capital cost of implementing a $\mu$ SR facility could be magnets. The existing MTA line components were chosen and are capable of supporting the optics required for a $\mu$ SR facility. The 6 new components have integrated quad strengths consistent with the current inventory of excess $200-\mathrm{MeV}$ quadrupoles, although the condition of specific magnets is not known. Further, they require magnetic field measurements and fiducial markers installed in order to align with any accuracy. Prior to installation of the MTA line, there were 16 green $200-\mathrm{MeV}$ quadrupoles and 1 without coils. Only 8 were used for the MTA line so 8 should remain and there are 2 Loma Linda quadrupoles which are somewhat stronger. Although muon campus has claimed all SQAs, there remains one spare SQA stored in the facility. The optics have not been optimized so the number may yet be reduced.

\section{Conclusions}

The Fermilab MuCool Test Area has the potential to be re-purposed into a world-class Muon Spin Resonance Facility. A near-term, limited facilitation would require only modest upgrades to the MTA experimental hall shielding and penetrations and the installation of magnetic components (from existing inventory) in the hall to transport full Linac intensity to the downstream 
high intensity absorber. The hall itself can accommodate a high-intensity muon production target viewed by a short, ultra-low energy, $\sim 30 \mathrm{MeV} / \mathrm{c}$, muon beamline and muon target station. A service building at ground level can accommodate all readout electronics and DAQ needs. A longer-term, more ambitious commitment to develop a dedicated $\mu$ SR facility into one competitive with existing facilities requires the experimental hall be converted to a target hall with a new highintensity beam absorber installed immediately downstream of a target pile. In this latter configuration up to three muon beamlines could be extended through the rollup door and shield wall into the large existing pit allowing access and avoiding high radiation exposure. For both scenarios breakthroughs in target design and engineering can achieve predicted factors of 2 in surface muon production. Relative to the limited approach, target design and removal of the transmission limitations (imposed by transport to the existing absorber) could potentially produce operational capabilities comparable to or even exceeding those of ISIS. In either case to go beyond the preliminary design concepts derived here requires directed and interactive efforts to model target parameters and production simultaneous with muon extraction, capture, and transport design as required to achieve an efficient high-performance facility. Specific issues such as groundwater contamination and air activation are issues which must be addressed by the design and cannot be resolved as an afterthought.

\section{Acknowledgements}

The $\mu$ SR analyses presented in this report would not have been possible without the extensive contributions in the past of C. Johnstone, I. Rakhno, N. Mokhov, ES\&H, and the many engineers whose extensive simulations and technical calculations were required to fully develop the MTA facility. Their work, much of which is unpublished, made possible the more detailed and specific technical evaluations of MTA capability discussed here.

\section{References}

[ 1] C. Johnstone, D.M. Kaplan, "Linac Test Facility Beam Properties”, MuCool Note 201, 2001

[2] S.L. Lee, S.H. Kilcoyne, R. Cywinski, "Muon Science (Muons in Physics, Chemistry and Materials", Institute of Physics Publishing, Bristol, 1999

[ 3] C. Johnstone, "Current Status of the MTA Beamline", MTA Beamline Review, 2012

[ 4] C.D. Moore, et al, "Design, Installation and Initial Commissioning of the MTA Beamline", Fermilab-Conf-09-616-ad, 2009

[ 5] Kamran Vaziri vaziri@fnal.gov, private communication, 2017

[ 6] Rick Baartman baartman@ @in12.triumf.ca, Syd Kreitzman syd@triumf.ca , private communication, 2017

[ 7] F. Berg, et al, "Target Studies for Surface Muon Production”, arXiv:1511.01288v2, 2016 
[ 8] E.W. Blackmore, et al, "Operating Experience with Meson Production Targets at TRIUMF, Proc. PAC-2005, Knoxville, 2005

[ 9] "Future Muon Sources", R. Cywinski, 2015, http://studylib.net/doc/5775639/future-muonsources---university-of-huddersfield

[10] A. Bungau, R. Cywinski, C. Bungau, "Simulations of surface muon production in graphite targets", PRSTAB 16, 2013

[11] A. Bungau, R. Cywinski, C. Bungau, "Target optimization studies for surface muon production", PRSTAB 17, 2014

[12] B.J. VerWest, R.A. Arndt, "NN Single Pion Production Cross Sections Below $1500 \mathrm{MeV",}$ Phys. Rev. C 25, 1982

[13] A. Bungau, et. al, "Geometry Optimization of the ISIS Muon Target, Proc. IPAC'10, Kyoto, 2010

[14] N. Mokhov, C. Johnstone, "MuCool Test Facility”, internal note, May, 2003

[15] I. Rakhno, C. Johnstone, N. Mokhov, "MuCool Test Facility Beam Absorber”, unpublished, 2003

[16] M. Gerardi, editor, "MuCool Facility Shielding Assessment”, 2010

[17] A. Lee, "The Temperature Study for Beam Dump used in Muon Cooling", attachment to shielding assessment, 2003

[18] D.R.F. Cochran, et al, "Production of Charged Pions by 730-MeV Protons from Selected Nuclei”, Phys. Rev. D 6, 1972

[19] K. Hagiwara, et al (Particle Data Group), "Review of Particle Physics", Phys. Rev. D 66, 2002

[20] E. Halkiadakis, "Particle Interactions with Matter", 2009, https://physicslearning2.colorado.edu/tasi/tasi_2009/Attachments/Halkiadakis_03_TASI_day3_s chool.pdf

[21] A. Cassacchia, et al, "Investigation of Emittance Analysis Methods Using the MuCool Test Area Beamline", submitted to NCUR2017, Memphis, TN, 2017

[22] I. Rakhno, C. Johnstone, "Radiation Shielding Calculations for MuCool Test Area at Fermilab", Fermilab-TM-2248, 2004

[23] I. Rakhno, C. Johnstone, "Radiation Shielding Issues for MuCool Test Area at Fermilab", Fermilab-TM-2305, 2005

[24] I. Rakhno, C. Johnstone, N. Mokhov, "MuCool Test Facility Beam Absorber", unpublished, 2003 
[25] "Fermilab Radiological Control Manual”, Article 236, http://www-esh.fnal.gov/FRCM

[26] N. Mokhov, C. Johnstone, "MuCool Test Facility”, internal note, 2003

$\Omega$ 